\title{
Spectral-based Simulations of Particle-laden Turbulent Flows *
}

\author{
K. Sengupta ${ }^{\text {a }}$, B. Shotorban ${ }^{\text {b }}$, G. B. Jacobs ${ }^{\text {c }}$, F. Mashayek ${ }^{\mathrm{a}, *}$ \\ ${ }^{a}$ Department of Mechanical and Industrial Engineering \\ University of Illinois at Chicago, 842 West Taylor Street, Chicago, IL 60607 \\ ${ }^{\mathrm{b}}$ Department of Mechanical and Aerospace Engineering \\ The University of Alabama in Huntsville, Huntsville, AL 35899 \\ ${ }^{\mathrm{c}}$ Department of Aerospace Engineering \\ San Diego State University, 5500 Campanile Drive, San Diego, CA 92182
}

\begin{abstract}
In this paper, we discuss the application of spectral-based methods to simulation of particle-laden turbulent flows. The primary focus of the article is on the past and ongoing works by the authors. The particles are tracked in Lagrangian framework, while direct numerical simulation (DNS) or large-eddy simulation (LES) is used to describe the carrier-phase flow field. Two different spectral methods are considered: Fourier pseudo-spectral method and Chebyshev multi-domain spectral method. The pseudo-spectral method is used for the simulation of homogeneous turbulence. DNS of both incompressible and compressible flows with one- and two-way couplings are reported. For LES of particle-laden flows, two new models, developed by the authors, account for the effect of sub-grid fluctuations on the dispersed phase. The Chebyshev multi-domain method is employed for the authors' works on inhomogeneous flows. A number of canonical flows are discussed, including flow past a square cylinder, channel flow and flow over backward-facing step. Ongoing research on particle-laden LES of inhomogeneous flows is briefly reported.
\end{abstract}

Key words: Eulerian-Lagrangian, DNS, LES, pseudo-spectral, spectral-multidomain, homogeneous turbulence, inhomogenous flows, stochastic modeling, channel flow, backward-facing step

\footnotetext{
* To appear in IJMF Special Issue

* Corresponding author, mashayek@uic.edu
} 


\section{Introduction}

This article primarily reviews the work conducted in our group, while also discussing, where relevant, the works of other groups with less emphasis. We consider a dispersed phase of solid particles in a carrier gas flow. The dispersed particles are treated as mathematical points, which are tracked in a Lagrangian framework. The two-way coupling of the particles with the carrier phase is treated by particle-source-in-cell (PSIC) method. The carrier gas is treated either by direct numerical simulation (DNS) or large-eddy simulation (LES), in both of which a set of Eulerian equations describe the flow. This approach is referred to as the Eulerian-Lagrangian approach to particleladen flow simulations [1]. The carrier gas flow is discussed in the context of both homogeneous and inhomogeneous turbulence. Fourier pseudo-spectral and spectral element methods are used for solving the carrier phase equations.

Fourier pseudo-spectral is the natural method of choice for the homogeneous turbulence when the periodic boundaries exist in all three directions. In this method, the dependent variables are expanded in terms of a set of complex exponential basis functions. Using a Fast Fourier Transform (FFT) algorithm, all dependent variables are transformed to the Fourier space. In the process of transformation, the nonlinear terms are treated in the physical space and this is the reason to call this method "pseudo-spectral." The nonlinear terms which consist of simple products, can be evaluated in the Fourier space from convolution sums. However, it is more efficient to form these products in the physical space than in the spectral space. In fact, nonlinear terms lead to the formation of spectral modes which do not lie within the resolved range of wavenumbers. When such modes are discretized, they are aliased to modes which are within the resolved wavenumber range. This causes 'aliasing' errors to be associated with the numerical solution. Also, since the particles are tracked in the physical space, the source terms require treatment in the physical space. The aliasing errors are the predominant source of numerical errors in spectral methods. There are several schemes to eliminate the aliasing errors. These schemes are discussed in detail in [2].

A major drawback of the spectral method described above is that approximation of the equations using Fourier series dictates a simple geometry, which in practice means a cube or a rectangle. Another restriction is that parallelization of the pseudo-spectral method is immensely dependent on the Fast Fourier Transform (FFT) routines and this creates some limitations depending on the computer architecture and the size of the problem. The computation

of expensive high Reynolds number flows requiring large degrees of freedom and parallel computing is therefore difficult.

It is clear that a scheme other than the traditional Fourier spectral method 
has to be considered for large-scale flow simulation in complex geometries. Several low order alternatives are of course available, such as finite volume, finite element and finite difference methods, but the consensus is that highorder methods are required to perform high-fidelity, efficient DNS. High-order methods require fewer degrees of freedom, and long-time integration in DNS is accurate, since high-order methods have small dispersion errors. Essentially, two suitable high-order schemes have come forth, including compact finite difference (FD) schemes with spectral-like resolution [3], and spectral/ $h p$ element (SE) type schemes [4-6].

FD is relatively easy to implement and program on structured grids. The application of boundary conditions in high-order FD, however, is complicated by the overlapping nature of the stencil. The use of high-order FD for complex geometry computation requires large multi-block structured grid, that is not trivial to implement. First, generation of grids of high-quality, essential to preserve the favorable characteristics of the method, with multi-block meshing is labor intensive and not always consistent when establishing grid convergence. Second, parallel codes are not optimal, since the overlap in FD stencil leads to a relatively large amount of data that needs to be exchanged between blocks on different processors. This reduces the applicability and efficiency of FD codes.

In SE the computational domain is divided into elements that are non-overlapping, providing a flexible meshing, easy boundary condition implementation, and highly parallel coding. In each element the solution values are approximated by an orthogonal polynomial (mostly Chebyshev or Legendre polynomial), which are also used in channel flow simulations with a single domain spectral method (e.g. [7-12]). Spectral convergence is achieved in SE by simply increasing the degree of the polynomial without widening the discretization stencil and the overlap like in FD. So, in addition to the mesh flexibility, SE is spectrally accurate, and establishing grid convergence is consistent and simple.

Within the broad class of spectral element methods, one should distinguish between continuous and discontinuous SE. In the continuous SE [4,5], connectivity between the elements is established by global assembly of local mass and stiffness matrices into one large matrix-vector formulation similar to finite element methods. This assembly is rather expensive and not very convenient for parallel implementation. In discontinuous spectral element methods [6], connectivity between elements is achieved by forcing the convective and diffusive fluxes to be continuous at the local element boundary only, while the solution is allowed to be discontinuous over element boundaries. The discontinuous method is local. Therefore, discontinuous SE combines all the desired features for high-fidelity DNS, including,

- high accuracy, requiring few degrees of freedom to resolve the smallest scale

- small dispersion error and long time accuracy 
- flexible meshing for complex geometries

- high parallelization capability

- ease of implementation

Compared to FD, SE is a relatively young method. Since its inception in the early eighties [13], much research has focused on establishing basic analysis and development. In recent years, DNS with the method has gained popularity for its favorable properties as described above.

Large-eddy simulation has proven to be a viable technique for the computation of unsteady turbulent flows with large coherent structures in complex flow applications. At moderate Reynolds numbers, LES is more economical and/or accurate than other established computational techniques such as DNS and Reynolds-average Navier-Stokes (RANS) methods. DNS solves the governing equations without any turbulence modeling. An accurate description of the Eulerian flow field with DNS enhances the accuracy of prediction of the dispersed phase. However, DNS requires excessive computational cost to resolve the turbulence scale range which increases with increase in geometric complexity and Reynolds number. Therefore, DNS of particle-laden flows are limited to low Reynolds numbers and simple configurations. In RANS, on the other hand, no attempt is made to resolve any of the turbulent motion, rather the net effect of all the scales on the mean flow is modeled. While this reduces computational cost and makes RANS feasible for complex engineering applications, it also prevents the method from adequately capturing the true dynamics of the flow. For particle-laden flows, in addition to fluid turbulence, models for particle dispersion due to turbulence are required. LES bridges the gap between DNS and RANS and efficiently computes flows in moderately complex geometries at mid range Reynolds numbers found in many real applications. LES is based on scale separation in turbulent flows: the large scales, which are anisotropic and sensitive to boundary conditions are computed directly as in DNS, while the small scales that are more isotropic and universal are modeled. The modeling of the small scales reduces the computational cost, while the computation of the large scales provides detailed flow field information. Thus, LES permits a more accurate accounting of particle turbulence interaction than RANS. We have performed LES of particle-laden homogeneous turbulence using pseudo-spectral method and of inhomogeneous flows using a spectral multidomain method. One of the major challenges in particle-laden LES is to model the effect of sub-grid scale motion on particles. These effects assume importance when substantial energy resides in the sub-grid scales and/or when particle time constants are small.

Particle-source-in-cell is an efficient method for incorporating two-way coupling in particle-laden flow simulations. In this article, we briefly discuss a high-order PSIC method that incorporates an efficient and accurate particle mover algorithm combined with a smooth weighting-function-based particle 
redistribution scheme.

The paper is organized as follows: First, we describe the governing equations for the carrier and dispersed phases. Next, we report our works on DNS and LES of particle-laden homogeneous turbulence using pseudo-spectral method. Then, we discuss particle-laden inhomogeneous flows, where a spectral multidomain method is used for simulation of the carrier phase. Again, both DNS and LES treatments of the carrier phase are considered. Finally, we draw some general conclusions on the use of spectral-based methods for simulation of particle-laden turbulent flows.

\section{Governing equations}

\subsection{Carrier phase}

In the majority of the equations considered in this paper the carrier phase is modeled as compressible and viscous fluid. The governing equations for the carrier phase flow are the conservation statements for mass, momentum and energy. They are presented in non-dimensional, conservative form with Cartesian tensor notation,

$$
\begin{aligned}
& \frac{\partial \rho}{\partial t}+\frac{\partial\left(\rho u_{j}\right)}{\partial x_{j}}=f_{m}, \\
& \frac{\partial\left(\rho u_{i}\right)}{\partial t}+\frac{\partial\left(\rho u_{i} u_{j}+p \delta_{i j}\right)}{\partial x_{j}}=\frac{\partial \sigma_{i j}}{\partial x_{j}}+f_{u_{i}}, \\
& \frac{\partial(\rho e)}{\partial t}+\frac{\partial\left[(\rho e+p) u_{j}\right]}{\partial x_{j}}=-\frac{\partial q_{j}}{\partial x_{j}}+\frac{\partial\left(\sigma_{i j} u_{i}\right)}{\partial x_{j}}+f_{e},
\end{aligned}
$$

where $\rho, u_{i}, p, \rho e$ are the density, velocity in the $i^{\text {th }}$ coordinate direction, thermodynamic pressure, and total energy per unit volume. The total energy, viscous stress tensor, $\sigma_{i j}$, and heat flux vector, $q_{j}$, are, respectively, given as

$$
\begin{aligned}
\rho e & =\frac{p}{\gamma-1}+\frac{1}{2} \rho u_{k} u_{k}, \\
\sigma_{i j} & =\frac{\mu}{R e_{f}}\left(\frac{\partial u_{i}}{\partial x_{j}}+\frac{\partial u_{j}}{\partial x_{i}}-\frac{2}{3} \frac{\partial u_{k}}{\partial x_{k}} \delta_{i j}\right), \\
q_{j} & =-\frac{\mu}{(\gamma-1) R e_{f} \operatorname{Pr}_{f} M_{f}^{2}} \frac{\partial T}{\partial x_{j}} .
\end{aligned}
$$


The source/sink terms $f_{m}, f_{u_{i}}$, and $f_{e}$ appearing in the governing equations represent the integrated effects of the dispersed phase mass, momentum and energy exchange with the carrier phase. These terms are considered only in our homogeneous flow simulations. The reference Reynolds number $R e_{f}$ is based on the reference density $\rho_{f}^{*}$, velocity $U_{f}^{*}$, length $L_{f}^{*}$, and molecular viscosity $\mu_{f}^{*}$ and is given by $R e_{f}=\rho_{f}^{*} U_{f}^{*} L_{f}^{*} / \mu_{f}^{*}$. $P r_{f}=\mu_{f}^{*} c_{p} / k^{*}$ is the reference Prandtl number. The superscript $*$ denotes dimensional quantities. The above equation set is closed by the equation of state,

$$
p=\frac{\rho T}{\gamma M_{f}^{2}},
$$

where $M_{f}$ is the reference Mach number, taken to be 1 in this work and $T$ is the temperature.

\subsubsection{LES formulation}

In LES, the governing equations for the carrier phase are the filtered compressible Navier-Stokes equations. By applying a spatial low-pass (in frequency domain) convolution filter to the Navier-Stokes equations, the turbulence scales are separated. The filter in physical space is represented by the following convolution product,

$$
\bar{f}(\boldsymbol{x}, t)=\int_{\Omega} f\left(\boldsymbol{x}^{\prime}, t\right) G\left(\boldsymbol{x}-\boldsymbol{x}^{\prime}\right) d \boldsymbol{x}^{\prime},
$$

where $G$ is the filter kernel and $\Omega$ represents the flow domain. We apply the Favre, density weighted filtering operation, typical for LES of compressible turbulent flow,

$$
\widetilde{f}=\frac{\overline{\rho f}}{\bar{\rho}},
$$

where overbar denotes the filtering operation. Applying this filter yields the following filtered conservation equations,

$$
\begin{aligned}
& \frac{\partial \bar{\rho}}{\partial t}+\frac{\partial\left(\bar{\rho} \widetilde{u}_{j}\right)}{\partial x_{j}}=0 \\
& \frac{\partial\left(\bar{\rho} \widetilde{u}_{i}\right)}{\partial t}+\frac{\partial\left(\bar{\rho} \widetilde{u}_{i} \widetilde{u}_{j}+\bar{p} \delta_{i j}\right)}{\partial x_{j}}=\frac{\partial \widetilde{\sigma}_{i j}}{\partial x_{j}}-\frac{\partial \tau_{i j}^{s g s}}{\partial x_{j}}+\frac{\partial\left(\bar{\sigma}_{i j}-\widetilde{\sigma}_{i j}\right)}{\partial x_{j}},
\end{aligned}
$$




$$
\begin{gathered}
\frac{\partial(\overline{\rho e})}{\partial t}+\frac{\partial\left[(\overline{\rho e}+\bar{p}) \widetilde{u}_{j}\right]}{\partial x_{j}}=-\frac{\partial \widetilde{q}_{j}}{\partial x_{j}}+\frac{\partial\left(\widetilde{\sigma}_{i j} \widetilde{u}_{i}\right)}{\partial x_{j}}-\frac{1}{(\gamma-1) M_{f}^{2}} \frac{\partial q_{j}^{s g s}}{\partial x_{j}}+(12 \\
\frac{\partial\left(\bar{q}_{j}-\widetilde{q}_{j}\right)}{\partial x_{j}}+\frac{\partial\left(\tilde{u}_{j}\left[\bar{\sigma}_{j k}-\widetilde{\sigma}_{j k}\right]\right)}{\partial x_{k}}+\frac{1}{2} \frac{\partial}{\partial x_{j}}\left[\bar{\rho}\left(\widetilde{u_{k} u_{k} u_{j}}-\tilde{u}_{k} \tilde{u}_{k} \tilde{u}_{j}-\tau_{k k}^{s g s} \tilde{u}_{j}\right)\right] .
\end{gathered}
$$

The filtering leads to several terms, in Eqs. (11) and (12), that require closure. $\tau_{i j}^{s g s}$ is the sub-grid scale stress tensor and $q_{j}^{s g s}$ is the sub-grid turbulent heat flux. These terms physically represent the effect of the unresolved (sub-grid) scales on the resolved scales. The second unclosed term in the filtered momentum equation is $\left(\bar{\sigma}_{i j}-\widetilde{\sigma}_{i j}\right)$, which results from Favre filtering of the viscous stresses. The filtered energy equation has three more unclosed terms in addition to the sub-grid heat flux: the term $\frac{\partial\left(\bar{q}_{j}-\widetilde{q}_{j}\right)}{\partial x_{j}}$ which results from Favre filtering of the diffusive heat flux; the term $\frac{\partial\left(\tilde{u}_{j}\left[\bar{\sigma}_{j k}-\widetilde{\sigma}_{j k}\right]\right)}{\partial x_{k}}$ which is analogous to the sub-grid scale viscous dissipation; and finally the divergence of turbulent diffusion, $\frac{1}{2} \frac{\partial}{\partial x_{j}}\left[\bar{\rho}\left(\widehat{u_{k} u_{k} u_{j}}-\tilde{u}_{k} \tilde{u}_{k} \tilde{u}_{j}-\tau_{k k}^{s g s} \tilde{u}_{j}\right)\right]$.

The unclosed terms in the filtered equations require modeling. The term $\left(\bar{\sigma}_{i j}-\right.$ $\left.\tilde{\sigma}_{i j}\right)$ is usually neglected $[14,15]$. In spectral element based simulations the subgrid term $\tau_{i j}^{s g s}=\bar{\rho}\left(\widetilde{u_{i} u_{j}}-\widetilde{u}_{i} \widetilde{u}_{j}\right)$ is usually modeled using the modification of the Germano model [16] for compressible flows [17]. The expression for $\tau_{i j}^{\text {sgs }}$ is accordingly given as

$$
\tau_{i j}^{s g s}=-2 C_{s} \bar{\triangle}^{2} \bar{\rho}|\widetilde{S}|\left(\widetilde{S}_{i j}-\frac{1}{3} \widetilde{S}_{m m} \delta_{i j}\right)+\frac{1}{3} \tau_{k k}^{s g s} \delta_{i j} .
$$

In compressible flow, the trace of the sub-grid stress tensor $\tau_{k k}^{s g s}$ cannot be included in the modified pressure, and therefore has to be modeled independently. Various models of $\tau_{k k}^{s g s}$ have been proposed (see $[18,19]$ ). However, previous studies $[20,21]$ have demonstrated that there is no difference in the LES results at low Mach number when $\tau_{k k}^{s g s}$ is neglected and in certain cases (see [21]) the SGS model that neglects $\tau_{k k}^{s g s}$ is more stable. Therefore, in LES of low Mach number flow, neglecting the trace of sub-grid stress tensor does not introduce large errors and is often beneficial towards numerical stability. The dynamic procedure gives a local time-dependent estimate of $C_{s} \bar{\triangle}^{2}$, which is updated at each time iteration. It is worthwhile to note that the dynamic procedure computes the Smagorinsky length scale $C_{s} \bar{\triangle}^{2}$ directly without the need to specify the grid filter width $\bar{\triangle}$. This is advantageous in the context of spectral element method, where it is difficult to provide a general expression for the filter width $\bar{\triangle}$.

The sub-grid term

$$
q_{j}^{s g s}=\bar{\rho}\left(\widetilde{T u_{j}}-\widetilde{T} \widetilde{u}_{j}\right)
$$


is usually modeled using the eddy-diffusivity hypothesis and a turbulent Prandtl number. The modeled expression is,

$$
q_{j}^{s g s}=\frac{\bar{\rho} C_{s} \bar{\triangle}^{2}|\widetilde{S}|}{P r_{t}} \frac{\partial \widetilde{T}}{\partial x_{j}} .
$$

The turbulent Prandtl number $P r_{t}$ is evaluated using a dynamic procedure analogous to the one used for computing the sub-grid viscosity. A priori analysis of the magnitude of various terms in the filtered energy equation by Vreman et al. [14] has shown that the fourth and fifth terms on the right-hand side of Eq. (12) are small compared to the sub-grid heat flux vector and can be neglected, especially at low and moderate Mach numbers. Finally, the last term in the filtered energy equation (12) is similar to turbulent diffusion of sub-grid scale kinetic energy and its contribution is again small compared to other sub-grid terms (see [22]).

The dynamic procedure requires the definition of an explicit, low pass filter for the test filtering operation. Spectral filtering can be constructed using either discrete polynomial transform (DPT) or interpolant-projection (see [23]) over each element. DPT filtering can be conveniently applied for methods with modal basis. For methods with nodal basis, the solution has to be first transformed to modal basis before the DPT filter can be applied. DPT filtering has been used in the discontinuous Galerkin LES methodology developed by the authors [24]. Projection filtering on the other hand can be constructed directly on the nodal basis. Since it does not require an extra transformation, interpolant-projection filtering is more efficient than DPT for methods with nodal basis. This kind of filtering was introduced for a spectral multidomain LES method in [25].

\subsection{Dispersed phase}

The non-dimensional Lagrangian equations governing the position $y_{i}$, velocity $v_{i}$, and temperature $T_{p}$ of each spherical particle, of radius $a_{p}$, mass $m_{p}$, and specific heat coefficient $c_{p}$, in the carrier flow field can be written as

$$
\begin{aligned}
& \frac{d y_{i}}{d t}=v_{i}, \\
& m_{p} \frac{d v_{i}}{d t}=F_{i}, \\
& m_{p} c_{p} \frac{d T_{p}}{d t}=Q,
\end{aligned}
$$


where $F_{i}$ denotes the summation of all the forces acting on the particle and $Q$ is the net rate of heat transfer to the particle. Equation (18) is written by assuming that the time scale of thermal conduction inside the particle is much shorter than the time scale of thermal convection outside, i.e. for small particle Biot numbers. For such a particle, temperature variation inside the particle can be neglected and thus the particle temperature $T_{p}$ can be considered uniform.

The problem of predicting the forces on a particle moving in a viscous fluid has been studied for more than 150 years since Stokes first obtained, in the year 1851, the drag force on a sphere in creeping flow condition. Over the years different equations have been proposed and used. Historical background and a detail account on the relevant works are available in review articles by Michaelides [26], Michaelides and Feng [27] and Gouesbet et al. [28]. Here we describe the equations which have been used in our work. For a more comprehensive description the reader is referred to the review by Mashayek and Pandya [1].

Oseen [29] proposed an equation for particle motion in shear flows. The equation popularly known as the BBO equation is presented in its non-dimensional form,

$$
\begin{gathered}
\frac{d v_{i}}{d t}=\frac{C_{D}}{S t}\left(u_{i}-v_{i}\right)+\frac{0.2 C_{H}}{(\epsilon S t)^{1 / 2}} \int_{t_{0}}^{t} \frac{d\left(u_{i}-v_{i}\right) d \tau}{\sqrt{t-\tau}} d \tau+ \\
C_{A} \frac{1}{\epsilon} \frac{d\left(u_{i}-v_{i}\right)}{d t}+\frac{1}{\epsilon} \frac{D\left(u_{i}\right)}{D t}+\frac{0.727}{(\epsilon S t|\vec{\Omega}|)^{1 / 2}}\left(u_{i}-v_{i}\right) \times \overrightarrow{\Omega_{f}}
\end{gathered}
$$

The first term represents the Stokesian drag based on particle slip velocity; the second term is the Basset history force, resulting from the temporal development of the particle wake and is important when the time scales of fluid acceleration are on the order of convection over particle surface; the third term is the added mass force; the fourth term is the result of acceleration of the local fluid element also referred as the stress-gradient effect; finally, the last term denotes the Saffman lift force. $\overrightarrow{\Omega_{\mathrm{f}}}$ is the fluid vorticity at the particle location. The coefficients $\mathrm{C}_{\mathrm{H}}$ and $\mathrm{C}_{\mathrm{A}}$ appearing in the above equation are given as,

$$
C_{H}=2.88+\frac{3.12}{\left(1+A_{c}\right)^{3}},
$$

and

$$
C_{A}=1.05-\frac{0.066}{A_{c}^{2}+0.12},
$$


where $A_{c}$ is the relative acceleration factor, given by

$$
A_{c}=\frac{|\vec{u}-\vec{v}|^{2} / d_{p}}{|d(\vec{u}-\vec{v}) / d t|}
$$

In Eq. 19, $\epsilon$ is the ratio of particle to fluid densities,

$$
\epsilon=\frac{\rho_{p}}{\rho_{f}}
$$

and the Stokes number, $S t$ is defined as the ratio of the particle response time, $t_{p}$, to the characteristic flow time, $t_{f}$,

$$
S t=\frac{t_{p}}{t_{f}}=\frac{\rho_{p}^{*} d_{p}^{* 2} U_{f}^{*}}{18 \mu^{*} L_{f}^{*}}=\frac{\rho_{d} d_{p}^{2} R e_{f}}{18} .
$$

The drag coefficient $\mathrm{C}_{\mathrm{D}}$ is given by,

$$
C_{D}=1+0.15 R e_{p}^{0.687}
$$

Michaelides and Feng [30,27] derived an expression for $Q$, (see Eq. (18)), in an unsteady flow and temperature field for a rigid sphere with high thermal conductivity and at low Peclet number. A simplified form of their equation is generally used in practical simulations. Here, only the convective heat transfer from the fluid phase to the particle is accounted for, while the effects of curvature, added mass and history force are neglected. This simplified form is known as the modified Michaelides-Feng (MMF) equation, whose non-dimensional form is presented below,

$$
\frac{d T_{p}}{d t}=\frac{N u}{3 \operatorname{Pr} S t}\left(T_{f}-T_{p}\right)
$$

where $T_{f}$ is the temperature of the carrier fluid at the location of the particle. The Nusselt number $N u$ for the spherical particle is given by Ranz-Marshall correlation [31] and is written as

$$
N u=2+0.6 \operatorname{Re}_{p}^{0.5} \operatorname{Pr}^{0.33} \quad \forall \quad R e_{p}<5 \times 10^{4}
$$

where $\operatorname{Pr}$ is the Prandtl number for the carrier fluid phase. The particle Reynolds number, $R e_{p}$ is given by,

$$
R e_{p}=R e_{f} d_{p}|\vec{u}-\vec{v}|
$$




\section{Homogeneous turbulence}

In homogeneous turbulence, the fluctuating quantities are statistically homogenous meaning that their average properties are independent of the position in the flow. The mean velocity gradient $A_{i j}=\partial\left\langle u_{i}\right\rangle / \partial x_{j}$ may be non-zero but a function of time only [32]. Due to the simplicity of the flow configuration in homogeneous turbulence, while retaining the rich turbulence physics, its simulation is widely performed. Complex physical phenomena, such as turbulence-wall interaction, are not a concern for simulation in homogeneous configurations. Therefore, in the presence of other physics such as particleturbulence interaction, one does not need to concern about wall interference when the focus of research is to study, for example, the collision of particles.

In homogeneous turbulence, the instantaneous velocity $u_{i}$ of the carrier phase is decomposed as

$$
u_{i}=A_{i j}(t) x_{j}+u_{i}^{\prime} .
$$

The periodicity of boundary conditions (necessary for application of Fourier spectral method) cannot be directly applied on the resulting equations for the fluctuating values due to the spatial variation of the mean velocity. One possibility to tackle this problem is to use a coordinate system that moves with the mean velocity. This coordinate transformation has been first used in the numerical simulation of single-phase homogeneous flow by Rogallo [33]. The transformation has been introduced for the first time in solving the linearized problem of turbulence [34] and reads

$$
\xi_{i}=B_{i j}(t) x_{j},
$$

where $B_{i j}$ satisfies the equation

$$
\dot{B}_{i j}+B_{i k} A_{k j}=0 .
$$

Replacing from (29)-(31) in (1)-(3), the governing equations for the compressible flow can be described as

$$
\begin{aligned}
& \frac{\partial \rho}{\partial t}+A_{i i} \rho+B_{i j} \frac{\partial}{\partial \xi_{i}}\left(\rho u_{j}^{\prime}\right)=0, \\
& \frac{\partial}{\partial t}\left(\rho u_{i}\right)+A_{j j} \rho u_{i}^{\prime}+A_{i j} \rho u_{j}^{\prime}+B_{l i} \frac{\partial}{\partial \xi_{l}}\left(\rho u_{i}^{\prime} u_{j}^{\prime}\right)=
\end{aligned}
$$




$$
\begin{aligned}
& B_{l j} \frac{\partial}{\partial \xi_{l}}\left[-p \delta_{i j}+\frac{\mu}{R e_{f}}\left(B_{n i} \frac{\partial u_{j}^{\prime}}{\partial \xi_{n}}+B_{n j} \frac{\partial u_{i}^{\prime}}{\partial \xi_{n}}\right.\right. \\
& \left.\left.-\frac{2}{3} B_{n k} \frac{\partial u_{k}^{\prime}}{\partial \xi_{n}} \delta_{i j}\right)+\frac{\mu}{R e_{f}}\left(A_{i j}+A_{j i}-\frac{2}{3} A_{k k} \delta_{i j}\right)\right]+f_{u_{i}}, \\
& \frac{\partial}{\partial t}(\rho \phi)+A_{j j} \rho \phi+A_{i j} \rho u_{i}^{\prime} u_{j}^{\prime}=A_{i j} \sigma_{i j} \\
& +B_{k i} \frac{\partial}{\partial \xi_{k}}\left(\sigma_{i j} u_{j}^{\prime}-\rho e u_{i}^{\prime}+\frac{\mu}{(\gamma-1) R e_{f} \operatorname{Pr}_{f} M_{f}^{2}} B_{l i} \frac{\partial T}{\partial \xi_{l}}\right)+f_{e},
\end{aligned}
$$

where

$$
\begin{aligned}
& \sigma_{i j}=-p \delta_{i j}+\frac{\mu}{R e_{f}}\left(\frac{\partial u_{i}^{\prime}}{\partial \xi_{l}} B_{l j}+\frac{\partial u_{j}^{\prime}}{\partial \xi_{l}} B_{l i}-\frac{2}{3} \frac{\partial u_{k}^{\prime}}{\partial \xi_{l}} B_{l k} \delta_{i j}\right) \\
& +\frac{\mu}{R e_{f}}\left(A_{i j}+A_{j i}-\frac{2}{3} A_{k k} \delta_{i j}\right)
\end{aligned}
$$

and $\phi=p /(\gamma-1)+\frac{1}{2} \rho u_{i}^{\prime} u_{i}^{\prime}$. It is noted that $f^{\xi}\left(\xi_{i}, t\right)=f\left(B_{i j}^{-1} \xi_{j}, t\right)$ and in the above equations $\xi$ is suppressed to avoid the complexity of the notation.

To derive the dispersed phase equations for homogeneous turbulence, first the deviation of the particle velocity from the local mean velocity of the carrier phase is defined

$$
v_{i}^{\prime}=v_{i}-A_{i j} y_{j}
$$

where $y_{j}$ is the position of particle in the physical domain. Using (30), the equations of particle in the transformed domain are derived as

$$
\begin{aligned}
& \frac{d \zeta_{i}}{d t}=B_{i j} v_{j}^{\prime}, \\
& \frac{d v_{i}^{\prime}}{d t}=\frac{C_{D}}{S t}\left(u_{i}^{\prime}-v_{i}^{\prime}\right)-A_{i j} v_{j}^{\prime},
\end{aligned}
$$

and the temperature equation is unchanged.

The homogeneous isotropic turbulence, or briefly called the isotropic turbulence, is the simplest turbulence configuration studied by DNS and LES. In the isotropic turbulence, $A_{i j}=0$ and the velocity and temperature fields are statistically invariant under translations, rotations and reflections of the coordinate system [32]. The isotropic turbulence could be decaying or (forced) 
stationary. Due to the dissipation, turbulence cannot sustain itself in the decaying isotropic turbulence so it is not statistically stationary. In order to generate a statistically stationary isotropic turbulence, the flow is forced at low wavenumbers meaning that through adding a source term to the righthand side of the momentum equation turbulent energy of the large scales is artificially generated at very low wavenumbers.

The homogeneous shear turbulence is the second most popular homogeneous configuration studied in particle-laden turbulent flows. In homogeneous shear turbulence the mean velocity gradient is $A_{i j}=\Gamma \delta_{1 i} \delta_{2 j}$ which means that the mean velocity vector has one component in the streamwise direction and it linearly varies in the cross-stream direction. Artificial forcing at larger scales is not necessary to maintain turbulence. However, without forcing, the simulation must be terminated at some time after which the homogeneity assumption breaks down. The reason for the lack of homogeneity in a long run is that, the large scales of turbulence grow in time and the computational domain size at some point in time becomes too small to capture these ever growing scales. Flow statistics in homogeneous shear turbulence always vary as a function of time.

\subsection{Carrier phase treated by DNS}

The use of DNS for particle-laden homogenous turbulent flows began more than three decades ago. Most of the DNS studies have been carried out to explore various physics, which would have been impossible otherwise in some cases. Also, DNS has been extensively used to generate data to test various RANS and LES models. In most of these studies, the carrier phase has been simulated by spectral methods.

The first DNS of particle-laden turbulence was performed by Riley and Patterson [35] for isotropic turbulence. Clustering or the so-called "preferential accumulation" of particles in turbulent flows was first observed in DNS by Squires and Eaton [36] and then further investigated in greater detail by others for isotropic configuration [37-43]. Figure 1 shows the accumulation of particles for various Stokes numbers (defined based on the Kolmogorov time scale) in a homogenous shear turbulence. The accumulation of particles is the most at $S t=1.6$ and the least at $S t=0.16$. At very small Stokes numbers, inertial particles behave similar to fluid particles; therefore, not a significant particle accumulation is seen at smaller Stokes numbers. With the increase of the Stokes number, inertial particles, due to the centrifugal effects, further spin out of vortical structures and accumulate in regions of high strain rate and low vorticity. At Stokes numbers around unity, the maximum accumulation

of particles occurs. With further increase of the Stokes number, the particles 
exhibit less accumulation since these high-inertia particles are less correlated to their local fluid flow. The anisotropy of particle accumulation is evident in Fig. 1.

To measure the preferential accumulation of particles and its anisotropy in the homogeneous shear turbulence, Radial Distribution Functions (RDFs) can be used [43]. A three-dimensional RDF is defined as the ratio of the number of particle pairs found at a certain separation distance to the expected number if the particles are uniformly distributed $[44,40]$. The three-dimensional RDF, for a total of $N_{\mathrm{p}}$ particles, is defined as

$$
g_{3 \mathrm{D}}\left(r_{i}\right)=\frac{P_{i} / V_{i}}{P / V}
$$

where $P=N_{\mathrm{p}}\left(N_{\mathrm{p}}-1\right) / 2$ is the total number of particle pairs; $P_{i}$ is the number of pairs within separation distance between $r_{i}-\Delta r / 2$ and $r_{i}+\Delta r / 2 ; \mathrm{V}$ is the total volume of the system and $\left.V_{i}=\frac{4}{3} \pi\left[\left(r_{i}+\Delta r / 2\right)^{3}-\left(r_{i}-\Delta r / 2\right)^{3}\right)\right]$ is the volume of the shell with a thickness of $\Delta r$ and a radius of $r_{i}$ at its middle. The two-dimensional RDF is defined as

$$
g_{2 \mathrm{D}}\left(r_{i}\right)=\frac{\tilde{P}_{i} / A_{i}}{\tilde{P} / A},
$$

where $\tilde{P}$ is the total number of particle pairs in a planar slice of small thickness; $\tilde{P}_{i}$ is the number of pairs within the planar slice with separation distances between $r_{i}-\Delta r / 2$ and $r_{i}+\Delta r / 2 ; A$ is the area of the planner slice and $A_{i}=\pi\left[\left(r_{i}+\Delta r / 2\right)^{2}-\left(r_{i}-\Delta r / 2\right)^{2}\right]$ is the area of the shell associated with the nominal separation distance $r_{i}$ within the planar slice. Note that three different planar RDFs can be computed by choosing mutually perpendicular planar slices and here we denote $g_{2 \mathrm{D}, i}$ to be the two-dimensional RDF with slice perpendicular to $x_{i}$ direction. Using $2 \mathrm{D}$ RDFs, the anisotropy of the preferential accumulation can be measured in homogeneous shear turbulence [43]. Figure 2 shows that at the scales smaller than the Kolmogorov length scales, particles are most accumulated in the streamwise direction (horizontal in Fig. 1) and they are least accumulated in the cross-stream directions.

The modulation of turbulence due to particles $[45,46]$ as well as the collision and coagulation of particles $[39,44]$, were also studied by DNS for isotropic turbulence. Sundaram and Collins [39] quantified the collision frequency correlation to the RDF and the relative velocity probability density function for various Stokes numbers. Reade and Collins [44] showed that these functions are also controlling the coagulation of particles.

Spectral method was also used for simulations of plain-strain and axisymmetric homogenous turbulent flows laden with particles $[47,48]$. The significant 
feature of these flows was the presence of a relative mean velocity between the two phases, unlike the homogeneous shear flow where the two phases had the same mean velocity $[49,50]$. This relative velocity led to an investigation of the "compressibility" of the dispersed phase, despite the incompressible carrier phase, as well as the crossing trajectories effect. The data published in $[47,48]$ could serve for validation of various two-phase models in capturing these important phenomena.

All of the above studies were carried out for isothermal cases treating carrier phase by DNS using spectral methods. There have also been several investigations carried out via spectral methods for non-isothermal particle-laden turbulent flows. Jaberi [51] showed that in non-isothermal, isotropic turbulence with stationary velocity and decaying temperature fields, the probability density function of the fluid temperature deviates farther away from a Gaussian distribution with the increase of the mass loading ratio. Jaberi and Mashayek [52] simulated the dispersed phase in forced isotropic turbulence. They found that the variance of the fluid and particle temperature increases as the mass loading ratio or the Prandtl number increases. The mechanism of heat transfer between the two phases in the presence of a mean temperature gradient in the decaying isotropic turbulence was also investigated by Sato et al. [53]. The results showed that the particle temperature and velocity were well correlated in the direction of the mean temperature gradient. Later, Shotorban et al. [54] studied the non-isothermal dispersion of particles in homogeneous shear turbulence. Their results indicated that the increase of the mass loading ratio or the particle time constant generally reduced the temperature variance and the magnitude of the turbulent heat flux of both carrier and dispersed phases. The data generated by DNS in this work was also used for verification of a probability density function model for the non-isothermal particle-laden turbulence [55].

Spectral simulation of homogeneous particle-laden turbulence was extended to compressible flows by Mashayek [56-61], while also considering evaporating/reacting droplets. The Mach number was chosen small to avoid dealing with shocks which are difficult to resolve with spectral method due to its low numerical diffusion. The results of the simulations were used to investigate the evolution of various terms in the transport equations for kinetic and internal energies as well as Reynolds stresses of both phases. The simulations were also useful to study the mechanisms of heat transfer between the particles and the carrier phase in both isotropic and homogeneous shear turbulence. The twoway coupling cases showed that the spectral method is capable of providing smooth results when using the PSIC method. 


\subsection{Carrier phase treated by LES}

The extent of published works in LES of particle-laden homogeneous turbulence is limited, as compared to DNS studies. To the best knowledge of the authors, Yeh and Lei $[62,63]$ were the first who implemented LES for the simulation of carrier phase to study particle dispersion in isotropic and homogeneous shear turbulent flows. Simonin et al. [64] used LES data to verify their two-fluid model. The LES model used in these studies were fixed-constant Smagorinsky in one-way coupling. Boivin et al. [65] conducted a priori test study for two-way coupling effect in isotropic turbulence. In all of these studies the effect of SGS on the particles was not considered and in the latter work, the effect of particles on the SGS was neglected in two-way coupling.

One of the main issues in implementing LES for treating the carrier phase is to model the effect of subgrid scales on particles. These effects cannot be neglected when the filtered energy is significant and/or the particle time constant is small [66]. The particles with larger time constants mainly interact with the large scales of turbulence. The effects of SGS on particles have been modeled through deterministic and stochastic approaches.

In the deterministic approach, the instantaneous velocities are reconstructed for the use in particle equations through defiltering [67-71]. Shotorban and coworkers $[68,69,71]$ proposed to use the Approximate Deconvolution (AD) [72] for defiltering. Kuerten and Verman [67] employed a defiltering technique in which the filtering inversion is carried out in the Fourier space for the streamwise and spanwise directions while the inversion is approximated by a Taylor series for the cross-stream direction. Although, defiltering can readily be performed, the major issue is that defiltering can be carried out only for the represented modes.

Shotorban [68] and Shotorban and Mashayek [73] proposed to use a Langevin type stochastic differential equation for evolution of particles when carrier phase is simulated by LES. A similar model was previously proposed for particles by Pozorski and Minier [74,75] and Minier and Peirano [76] when the carrier phase is simulated by RANS. Improved Langevin models were later proposed by Shotorban and Mashayek [73] and Berrouk et al. [77]. In the Langevin models, the seen fluid particle velocity in the Langevin equation is modeled as

$$
d u_{\mathrm{s} i}=A_{i} d t+B_{i j} d W_{j},
$$

where $A_{i}$ and $B_{i j}$ are drift and diffusion coefficients which are functions of $t, y_{i}, v_{i}$, and $u_{\mathrm{s} i}$. Shotorban and Mashayek [73] extended Langevin applications in particle-laden RANS [74-76] to LES using the stochastic differential 
equation employed to solve LES equations through Filtered Density Function approach [78],

$$
A_{i}=-\frac{1}{\rho_{f}} \frac{\partial p}{\partial x_{i}}+\frac{1}{\operatorname{Re}_{0}} \frac{\partial^{2} \bar{u}_{i}}{\partial x_{j} \partial x_{j}}-\frac{u_{\mathrm{s} i}-\bar{u}_{i}}{T_{L}^{*}} ; \quad B_{i j}=\sqrt{C_{i j} \epsilon},
$$

where $T_{L}^{*}$ and $\epsilon$ are an appropriate subgrid-scale time scale and the subgridscale dissipation rate, respectively. $C_{i j}$ are the model constants. The calculation of $T_{L}^{*}$ and $\epsilon$ and the accompanied model constants are given by Giquel et al. [78] for single-phase flows. This calculation and the constants were implemented by Shotorban and Mashayek [73] for the case of particle-laden flows and were recently modified by Berrouk et al. [77] to account for anisotropy of crossing-trajectory effects. A similar anisotropy extension had been proposed for RANS in [76].

To verify their proposed models, which were to account for the effect of SGS on particles, Shotorban and coworkers $[69,73,79,71]$ conducted LES of homogeneous turbulence with one-way coupling. The verification of their stochastic model was carried out in a decaying isotropic turbulent flow [73]. They showed that the stochastic model well predicts the results obtained by DNS for small particle time constants and with the increase of the particle time constant, the discrepancy between stochastic model and DNS results increases. Shotorban and Mashayek [79] improved their stochastic model predications by a modification. Their improved model took into account the high inertia effect of large particle time constants. The verification of approximate deconvolution for the reconstruction of the approximate instantaneous velocity for the use in the particle equations was performed in homogenous shear turbulence [79,71].

\section{Inhomogeneous turbulence}

\subsection{Carrier phase treated by DNS}

A complete review of the DNS with SE is outside the scope of the review here. Here, we focus on the DNS of compressible flow with discontinuous SE, that has received relatively little attention. In [80], the multi-domain spectral method was validated for simulation of compressible turbulent flows. The highorder Navier-Stokes solver is tested extensively for 2D and 3D gas dynamics problems [81-83,80,84], and proven itself efficient, accurate, and robust. It was shown that the method requires fewer degrees of freedom compared to lower-order methods to resolve the turbulence energy spectrum. In $[85,86]$, computations on a backward-facing step show its efficiency for computation in complex geometries. 


\subsubsection{Particle-Source-in-Cell Method}

Typically, when dealing with complex geometries, the carrier phase computation in conjunction with PSIC relies on simple Cartesian grid based methods [87]. Discretizion is performed with the low order (typically second order) finite-difference time-domain solver. The Cartesian grid is essential to the success of the PSIC method, since it yields an accurate two-way particle coupling with low levels of numerical noise. The Cartesian grid, however, severely limits the geometric flexibility. Moreover, the low order scheme restricts temporal and spatial accuracy. This issue causes considerable problems when considering large scale problems in complex geometries. In particular, significant dispersion errors appear unless prohibitively fine grids are used.

In on-going efforts, we are addressing these limitations by developing highfidelity PSIC based on a carrier-flow computation with spectral methods [88,89]. The spectral multi-domain method alleviates the short-comings encountered in the low-order methods for simulation of the carrier phase as discussed in Introduction. The challenges in developing PSIC based on the high-order multidomain method lie in a consistent two-way coupling, that preserves the favorable carrier-flow simulation characteristics in the two-way coupled PSIC simulation.

We have developed an efficient particle tracking algorithm that is consistent with the high-order multidomain method $[88,89]$. The particle tracking algorithm takes advantage of the mapping in the carrier flow solver, that maps each physical element in the grid to one square master element. By mapping the particle coordinate to the same master element, many parts of the particle algorithm can be simply performed in the mapped space; locating the particle's host cell simplifies to comparison of the mapped particle coordinate to the square's coordinates. A Lagrange interpolation on the structured, orthogonal grid within each master element determines the carrier phase flow at the particle location. Higher dimensional interpolation is easily performed on the local tensorial grid in each element. It is noted that a stable and accurate two-way coupled PSIC simulation requires the order of interpolation to match the order of the approximating polynomial within each element. In a one-way coupled simulation, where the particle motion is influenced by the carrier flow but not vice versa, a lower order Lagrange interpolation provides engineering accuracy, while saving computational time. From stability and accuracy considerations of a two-way coupled computation, it follows that the time integration scheme has to match the integration scheme used for the carrier flow. The developed algorithm handles complex particle boundary interaction by determining a levelset using a multi domain method. The precomputed levelset determines the distance and direction relative to the wall at each grid point. High-order interpolation of the levelset to the particle position then provides all the necessary information to implement elastic/inelastic interactions 
with complex boundaries.

The particle influence on the continuum is determined by redistribution of the mass, momentum and energy generated by the particle. Redistribution computations use large smooth weight functions. The smooth weighing prevents Gibbs oscillations that occurs in high-order interpolation of non-smooth functions. Compared to typical non-smooth redistribution schemes, this dramatically decreases the number of particles needed in a computational cell to produce low noise sources without filtering. Low-order redistribution usually requires additional filtering [90]. A large smooth Gaussian-like function $[89,91]$ per particle sums the particle's influence into the source terms in Eqs. (1)-(3). The high-order weight function reduces the numerical instabilities attributed to aliasing that is a result of the finite size of the particle cloud and non-linearity in the source terms.

\subsubsection{Particle-Laden Flow Computations}

We have studied several one-way coupled particle-laden flows with the highorder SE based DNS of the carrier phase. In [83], we studied the particle dispersion in the wake of a blunt body. The two-dimensional, shedding square cylinder flow was simulated on a grid with 196 elements. From a convergence study [85] it followed that an eighth-order approximation per element sufficed for a grid independent solution. The total number of required grid points was thus approximately 16,000. This is a significantly lower resolution requirement of around 70,000 points required for lower order simulations of the flow over the rectangular cylinder [92].

We identified a lock-in behavior of particles in the wake of the cylinder. The particles released behind the rectangle are shielded from the incoming flow by the cylinder. The periodically changing vertical direction of the flow behind the square leads to vertical transport of particles. The vertically transported particles accumulate at the rear corners of the square. Once a carrier phase vortex is shed off, the accumulated particles shed along with the vortex. Figure 3 shows the locked-in packages as observed downstream of the vortices in the wake's von Karman street. Particles were found to lock in between the shedding vortices when the particle response time is nearly equal to the time period of the vortex shedding in the wake, i.e. when $\phi$ defined by the ratio of the particle time constant to the shedding time period is of the order unity,

$$
\phi=\frac{t_{d}}{1 / f}=\frac{S t}{1 / S t r} \simeq 1,
$$

where $f$ is the shedding frequency and Str is the Strouhal number. It was determined that forces other than the Stokes drag, such as the Basset history 
and Saffman lift forces can blur the particle focusing in wake flows when the particle to fluid density ratio is less than 20 .

We studied particle-laden, unsteady, separated flow in [93]. We computed a non-confined or open backward-facing step carrier flow at a Reynolds number of $R e_{f}=1500$ based on the step height and the inflow velocity, and a Mach number of $M a=0.4$ based on the inlet velocity and the isothermal wall temperature. In Jacobs [85] this flow was studied extensively in two and three dimensions. The computational domain and grid are shown in Figure 4. The grid consists of 180 domains. On each subdomain a tenth-order approximation of the Navier-Stokes equations is projected using a nodal basis. The computational domain size is chosen to ensure a minimal blockage of the top inflow. At the left inflow boundary a uniform velocity is specified, while at the right outflow boundary a velocity profile is specified according to an experimental averaged turbulent boundary layer. The wall boundary conditions are no-slip and isothermal. The flow is initialized with the uniform inlet velocity.

In 2D, the the backward-facing step flow exhibits a quasi-turbulent, periodic shedding of a three-vortex system behind the step [94]. First, a vortex forms near the corner of the step. This vortex grows in strength and size as it is fed by the external flow. With increasing strength, the vortex increasingly pushes itself away from the wall, until it sheds off. The shedding occurs in an intimate three vortex interaction between the vortex at the step, the corner vortex behind the step, and the shed vortex (Fig. 5(a)).

The averaged flow pattern is quite regular with a typical recirculation region behind the step (Fig. 5(b)). A corner vortex is also present behind the step in the averaged flow field. The flow separation at the sharp edge of the step is trivial. The shear layer that emanates from the step corner reattaches behind the step. The non-trivial flow separation on the lower wall behind the step between the corner vortex and the recirculation sets the stage for a particle dispersion study.

The separation behavior is analyzed for several Stokes numbers that are based on a carrier flow time defined by the ratio of step height over the free stream velocity. Inertial particles with a Stokes number less than one, injected at the corner separation location are moved away from the wall by the separating carrier phase flow along a line that consequently moves with a shed vortex. This dispersion behavior is comparable to the moving separation behavior of separating fluid particles [95]. With the Stokes number increasing to one, the particles eject away from the wall to a lesser extent, until at $S t=1.0$ the particles are trapped at the corner separation location. When the Stokes number is larger, the particles (remarkably) start to separate away from the wall again. The particles, however, no longer move along with the shed vortex like the smaller Stokes number particles. Instead they separate into distinct material 
lines, whose origin remains fixed in time (Fig. 6). This separation behavior of particles is much like the fixed separation of fluid particles in moderately fluctuating unsteady separated flows [96]. In separating flow with large fluctuations, the delayed response of inertial particles to the fluid leads to inertial particles separating in a fixed manner, while the fluid particle separating line is moving. The Saffman lift force moves the particles away from the wall and pollutes the distinct character of the separation material line.

We further performed large-scale DNS of the three-dimensional transitional flow over the same backward-facing step geometry $[85,86]$ at $R e=3000$. A counter-current suction applied at the step corner was investigated to enhance mixing and dispersion of the particle-laden flow behind the step.

Periodic boundary conditions in the third dimension establish a periodic flow. For the computation to be stable and to capture the periodic structures, a minimum non-dimensional length of 6 was required. An eighth-order approximation converges the solution, compliant with the resolution requirements established in [80]. The total number of grid points for this computation is four million. This is a factor three improvement over the incompressible computation with a low order finite volume method employing 12 million grid points in [97]. Though the incompressible simulation showed good comparison of the averaged and turbulent stresses with the experiment, some discrepancies were observed. Moreover, no data on the dissipation rate was available, which is critical to establish a sufficient DNS resolution. The SE simulation was compressible, whereas the finite volume computations were incompressible. In addition to the difficulty in comparing compressible SE DNS with incompressible finite volume DNS, it is, for these reasons, difficult to directly compare the resolution of the SE and the finite volume.

The averaged flow statistics of the carrier phase showed excellent comparison with published experimental results on the incompressible flow at $M a=0.2$. At $M a=0.4$, the pressure-dilatation was observed to contribute significantly to the dissipation rate. The enhanced dissipation was believed to be responsible for the slightly shortened reattachment length of the recirculation bubble as compared to the incompressible flow experiment. The computed averaged velocities (Fig. 7) and the Reynolds stresses (Fig. 8) therefore show a slight shift as compared to the experimental data. When this shift (of $\Delta x=1$ ) is taken into account, the statistics are in excellent agreement (Fig. 8).

We investigated the dispersion of periodically-injected particles at the step corner. A limited understanding of this type of flow in liquid-fuel dump combustors motivated this study. Dump combustors are characterized by a suddenly changing or expanding geometry comparable to the backward-facing step. The dispersion mechanisms of injected fuel that atomizes into droplets in the recirculation zone (or "dump"), are complex and are not documented. 
Here, we report the first strides in our on-going studies.

Typically, droplets form upstream of the step. To investigate the dispersion, we therefore injected particles, as shown in Fig. 9, slightly upstream of the step corner at a streamwise location of $x=4.95$, which is upstream of and close to the step at $x=5.0$ [98]. Two release positions in the wall normal direction at $z=1.03$ and $z=1.10$ where considered. The first release position is inside the boundary layer at the step. The second release position is towards the outside of the boundary layer at the step. Particles are periodically released at their respective positions with a time interval of 0.25 . The simulations are run for $\mathrm{t}=16.0$, at which time snapshots of the particles are inspected. The particles' velocities are initialized with the fluid velocities at the particles' positions. Two particle Stokes numbers of $S t=0.1$ and 1.0 are considered.

In Figures 10 and 11 the particle snapshots for all cases are shown in side and top views, respectively. The (a) and (f) figures are reserved for pressure iso-contours of the carrier phase to identify the carrier phase flow structures at the time of the snapshot. From the side view of the non-manipulated BFS flow, large growing structures are observed in the transient unstable shear layer. The top view shows that the structures are nominally $2 \mathrm{D}$ up to the reattachment point of the shear layer. When the flow reattaches, the shear layer bursts, and the flow further downstream is mostly 3D and turbulent, recovering to a fully developed turbulent wall flow. A slight suction at the corner has a large impact on the flow. The shear layer, that is now almost immediately three dimensional, feeds a large turbulent recirculation structure behind the step.

The particles with a faster response time are increasingly captured in the recirculation region behind the step (Figs. 10 and 11). Particles with $S t=1$ injected outside the boundary layer are convected over the recirculation region. The employment of the countercurrent shear clearly enhances the mixing of the particles (right portions of Figs. 10 and 11). More particles are dumped as a result of countercurrent shear, and they tend to accumulate in the larger, more stable recirculation structure (as compared to the non-manipulated backwardfacing step flow) of the countercurrent shear flow. Large longitudinal flow structures were observed near the bottom wall in the recirculation area behind the corner. These structures transport particles upstream towards the step.

\subsection{Carrier phase treated by LES}

Spectral element methods are excellent candidates for LES of practical flows. The features that make them superior to low-order methods and/or single domain spectral schemes for DNS of practical flows, are equally beneficial in 
a LES context. However, there have been limited attempts to apply these methods towards LES. Spectral element filtering strategies have been studied in $[99,100,23,101,102]$. We have recently developed LES methodologies for compressible flows using two discontinuous spectral element methods [24,25]. Detailed description of the methodologies is beyond the scope of this review. The governing equations for LES of particle-laden compressible flows and the closure of the sub-grid terms, for the one-way coupling case are discussed in section 2 .

The dispersed phase equations are the same as those outlined in section 2.2. However, the fluid velocities in the equation for particles requires additional consideration. In flows where the effect of sub-grid scale (SGS) fluctuations on particle dispersion is expected to be negligible, the resolved scale velocity, which is directly available in LES simulation is used for calculating the particle slip as discussed in section 2. On the other hand, if the effect of SGS fluctuations is to be incorporated, then the sub-grid component is added to the resolved scale velocity for computing the particle slip (see section 3.2).

\subsubsection{LES of inhomogeneous shear flows}

All turbulent flows of practical interest are inhomogenous, where the inhomogeneity could arise from spreading of the flow into non-turbulent ambient (e.g. jets) or imposed by rigid flow boundaries (e.g. channel) or a combination of both (e.g. backward-facing step). LES studies of particle-laden inhomogeneous flows have focussed mostly on jets and channels.

Among wall bounded shear flows, the fully-developed turbulent channel flow has been studied most frequently, owing to its geometric simplicity, allowing the application of periodic boundary conditions in the streamwise and spanwise directions. Most of these works have used single domain spectral schemes or finite difference methods. Wang and Squires [103] studied particle-laden incompressible channel flow at two different Reynolds numbers. They considered one-way coupling between the phases, and the only forces acting on the particles were the drag and gravity. The subgrid stresses were modeled using a dynamic eddy-viscosity model. Particles with different time constants were considered and the dispersed phase statistics were compared with the DNS of Rouson and Eaton [104] and experiments of Kullick et al. [105]. The mean streamwise velocity compared well with the DNS for the particles with small time constants. However, second-order statistics showed considerable differences, possibly due to the coarse grid used for the carrier phase.

Armenio et al. [66] studied the effect of the sub-grid scale motion on the particle dynamics in a turbulent channel flow at $\mathrm{Re}_{\tau}=175$. They used a pseudospectral collocation method for solving the carrier phase equations. In order 
to study the effect of sub-grid scale velocity fluctuations on particle motion without the influence of modeling errors, they performed a priori analysis, where the tracer particles were advanced using the fully resolved velocity field from DNS and the filtered velocity field. In a second set of simulations they studied the effect of interpolation and subgrid modeling on particle motion in an actual LES. They concluded that at the Reynolds number considered, the statistics computed were not very sensitive to the subgrid velocity fluctuations, unless a significant portion of the energy was removed with a large filter. The use of the dynamic Smagorinsky model over the Smagorinsky model resulted in a much better dispersion statistics in their a posteriori analysis. They also studied the effect of including inertia of particles and observed that the inertial particles were less affected by sub-grid motions than were the tracer particles.

Recently Kuerten [70] performed LES of particle-laden turbulent channel flow using the dynamic Smagorinsky and approximate de-convolution model. FourierGalerkin method was used in the periodic directions while Chebyshev-collocation method was applied in the wall normal direction. Three different particle Stokes numbers and two Reynolds numbers were considered. For the low Reynolds number case, it was shown that the particle velocity fluctuations were underpredicted when filtered velocity available from LES was used in the particle equations. Better agreement was obtained with an inverse filtering model. The approximate deconvolution model used in the work gave better results than the dynamic eddy viscosity model. However, the difference between the two models was smaller for the high Reynolds number case.

Single-phase large-eddy simulations of channel flow using spectral element method have been performed by Blackburn and Schmidt [23] and more recently by the authors [25]. In [25], flow at two different Reynolds numbers, $\operatorname{Re}_{\tau}=180$ and $\operatorname{Re}_{\tau}=570$ were investigated. A sub-domain distribution of $10 \times 10 \times 16$ was employed in the streamwise, spanwise and wall normal directions for both Reynolds numbers. Two polynomial orders, $\mathrm{p}=6$ and $\mathrm{p}=8$ were used for $\operatorname{Re}_{\tau}=180$ and $\mathrm{Re}_{\tau}=570$, respectively. Figure 12 compares the mean velocity profile with the DNS of Moser et al. [106] and the experiment of Niederschulte et al. [107]. Good comparison with experiment and resolved DNS demonstrates that the LES methodology proposed in [25] is able to predict the flow. Therefore, the dispersed phase computation is started with the above stationary fluid flow field. The channel in vertically oriented as in the study by Wang and Squires [103] and Rouson and Eaton [104]. The Stokes number of the particles defined by Eq. (24) is 2.0, which corresponds to the $50 \mu \mathrm{m}$ diameter glass particles used in [103] and [104]. The particle forces taken into account are the Stokesian drag and gravity. Properties of the dispersed phase are obtained by following the trajectories of 200,000 particles. The particles are initially uniformly distributed over the channel. The boundary conditions for the dispersed phase include periodicity in the streamwise and spanwise directions and a specular reflection at the walls. The statistics are obtained 
by binning the particles in homogeneous boxes stacked along the wall normal direction. In figure 13, the mean streamwise velocity for the dispersed phase is compared with that from the DNS of Rouson and Eaton [104]. The mean velocity for the carrier phase is also shown in the figure. The LES result for particles are in good agreement with DNS for $\mathrm{z}^{+}>10$. The under-prediction $(\sim 5 \%)$ near the wall is because the near wall structures are not as accurately resolved in LES as in DNS and consequently the preferential concentration of particles in the near wall low speed streaks are lower in LES. The particles lead the fluid in the viscous sub-layer and in the bottom part of the buffer layer $\left(\mathrm{z}^{+}<12\right)$, while slightly lagging the fluid in the region above that. Calculation of second-order statistics for the dispersed phase is currently underway and will be reported in a future publication.

\section{Concluding remarks}

We have reviewed computational research on particle-laden turbulent flows using spectral methods for direct numerical simulation (DNS) and large-eddy simulation (LES) of the carrier phase in conjunction with particle-source-incell (PSIC) method for the dispersed phase.

Homogeneous turbulence computation with Fourier pseudo-spectral method has provided an idealized, but accurate carrier phase determination that has been the work horse for fundamental studies of particle-laden turbulent flows and the development of LES and Reynolds-average Navier-Stokes (RANS) models. In a large body of work, fundamental particle dispersion behavior, turbulence modification by particles, and particle-fluid interactions have been identified. A wealth of statistical data is available for the validation and improvement of particle-laden flow turbulence models developed in the frameworks of LES, RANS, and probability density function (PDF).

In a higher level of flow complexity that includes boundary effects, the particleladen, turbulent channel, jet and shear flows have been computationally studied with spectral or spectral-like methods. Despite the relative geometric simplicity (usually a rectangular domain), boundary effects have been shown to have large impact on the flow and the particle dispersion. Particle motion is significantly affected by the large coherent structures forming in these flows. Particle-wall interactions and the modeling thereof continue to pose a significant problem in LES simulation. A large amount of data is also available for these flow geometries.

The focus of computation of particle-laden flow in complex geometries, has been on the development of accurate spectral-like methods for simulation of particle-laden turbulent flows. In a series of papers, a spectral carrier-phase 
solver and an accurate particle-fluid coupling have been developed and validated. In two-dimensions the quasi-turbulent particle-laden flow over a square cylinder and a backward-facing step have been studied. Recently, the threedimensional particle-laden backward-facing step using a multidomain spectral method has also been computed.

Despite significant work, the near future in particle-laden compressible, turbulent flow research holds many challenges. The degrees of freedom in a complex geometry are of course unlimited. Computation of any complex geometry with DNS is and will be, for a foreseeable future, too computationally intensive despite significant computational resources. Therefor, more computationally affordable LES methods are eminent to further physical understanding and ability to analyze real particle-laden flows.

\section{Acknowledgements}

The authors wish to acknowledge the financial support of the National Science Foundation, the U.S. Office of Naval Research, and the Petroleum Research Fund. The computational resources were provided by the National Center for Supercomputing Applications and the San Diego Supercomputing Center.

\section{References}

[1] Mashayek, F. and Pandya, R. V. R., Analytical Description of Particle/Droplet-Laden Turbulent Flows, Progress in Energy and Combustion Science, 29(4):329-378 (2003).

[2] Canuto, C., Hussaini, M. Y., Quarteroni, A., and Zang, T. A., Spectral Methods in Fluid Dynamics, Springer-Verlag, New York, NY, 1987.

[3] Lele, S. J., Compact finite difference schemes with spectral-like resolution, J. Comp. Phys., 103:16-42 (1992).

[4] Karniadakis, G. E. and Sherwin, S. J., Spectral/hp Element Methods for CFD, Oxford University Press, New York, NY, 1999.

[5] Deville, M. O., Fischer, P. F., and Mund, E. H., High-Order Methods for Incompressible Fluid Flow, Cambridge University Press, 2002.

[6] Hesthaven, J. and Warburton, T., Nodal discontinuous Galerkin Methods: algorithms, analysis, and applications, Springer-Verlag, Berlin, 2008.

[7] Moin, P. and Kim, J., Numerical Investigation of Turbulent Channel Flow, J. Fluid Mech., 118:341-377 (1982).

[8] Ounis, H., Ahmadi, G., and McLaughlin, J. B., Dispersion and Deposition of Brownian Particles from Point Sources in a Simulated Turbulent Channel Flow, J. Colloid and Interface Science, 147(1):233-250 (1991). 
[9] Pedinotti, S., Mariotti, G., and Banerjee, S., Direct Numerical Simulation of Particle Behavior in the Wall Region of Turbulent Flows in Horizontal Channels, Int. J. Multiphase Flow, 18(6):927-941 (1992).

[10] Chen, M., Kontomaris, K., and McLaughlin, J., Dispersion, Growth, and Deposition of Coalescing Aerosols in a Direct Numerical Simulation of Turbulent Channel Flow, pp. 27-32, ASME, FED-Vol. 228, 1995.

[11] Rouson, D. W. I., Eaton, J. K., and Abrahamson, S. D., A Direct Numerical Simulation of a Particle-Laden Turbulent Channel Flow, Department of Mechanical Engineering Report TSD-101, Stanford University, Stanford, CA, 1997.

[12] Narayan, C., Lakehal, D., Botto, L., and Soldati, A., Mechanisms of particle deposition in a fully developed turbulent open channel flow, Phys. Fluids, 15(3):763-775 (2003).

[13] Patera, A. T., A Spectral Element Method for Fluid Dynamics - Laminar Flow in Channel Expansion, J. Comp. Phys., 54:468-488 (1984).

[14] Vreman, B., Guerts, B., and Kuerten, H., Subgrid-modeling in LES of compressible flow, Appl. Sci. Res., 54:191-203 (1995).

[15] Vreman, B., Guerts, B., and Kuerten, H., Large-eddy simulation of turbulent mixing layers, Journal of Fluid Mechanics, 339:357-390 (1997).

[16] Germano, M., Piomelli, U., Moin, P., and Cabot, W. H., A Dynamic SubgridScale Eddy Viscosity Model, Phys. Fluids A, 3(7):1760-1765 (1991).

[17] Moin, P., Squires, W., H., C. W., and Lee, S., A Dynamic Subgrid-Scale Model for Compressible Turbulence and Scalar Transport, Phys. Fluids A, 3:2746-2757 (1991).

[18] Yoshizawa, A., Statistical Theory for Compressible Turbulent Shear Flows, with the Application to Subgrid Modeling, Phys. Fluids, 29:2152-2164 (1986).

[19] Erlebacher, G., Hussaini, M. Y., Speziale, C. G., and Zang, T. A., Toward the Large Eddy Simulation of Compressible Turbulent Flows, J. Fluid Mech., 238:155-185 (1992).

[20] Squires, K. D., Dynamic subgrid scale modeling of compressible turbulence, Annual Research Brief, Stanford University, 1991.

[21] Vreman, A. W., Guerts, B. J., and Kuerten, J., Direct and large-eddy simulation I, Kluwer academic publisher, Netherlands, 1994.

[22] Santhanam, S., Lele, S. K., and Ferziger, J. H., A robust high-order compact method for large-eddy simulation, Journal of Computational Physics, 191:392-419 (2003).

[23] Blackburn, H. M. and Schmidt, S., Spectral element filtering techniques for large-eddy simulation with dynamic estimation, Journal of Computational Physics, 186:610-629 (2003). 
[24] Sengupta, K., Jacobs, G. B., and Mashayek, F., Large-eddy simulation using a disconitunous Galerkin spectral element method, AIAA Paper 07-0402, 2007.

[25] Sengupta, K., Jacobs, G. B., and Mashayek, F., Large-eddy simulation using a spectral multi-domain method, International Journal for Numerical Methods in Fluids, (2008), submitted.

[26] Michaelides, E. E., Review-The Transient Equation of Motion for Particles, Bubbles, and Droplets, Journal of Fluid Engineering, 119:233-247 (1997).

[27] Michaelides, E. E. and Feng, Z.-G., Analogies between the Transient Momentum and Energy Equations of Particles, Prog. Energy Combust. Sci, 22:147-162 (1996).

[28] Gouesbet, G. and Berlemont, A., Eulerian and Lagrangian Approaches for Predicting the Behaviour of Discrete Particles in Turbulent Flows, Prog. Energy Combust. Sci., 25:133-159 (1999).

[29] Oseen, C. W., Uber die Stokes'sche formel, und uber eine verwandte aufgabe in der hydrodynamik, Hydromechanik, 82:21-29 (1927).

[30] Michaelides, E. E. and Feng, Z.-G., Heat Transfer from a Rigid Sphere in a Nonuniform Flow and Temperature Field, Int. J. Heat Mass Transfer, 37:2069-2076 (1994).

[31] Ranz, W. E. and Marshall, W. R., Evaporation from Drops, Chem. Engng Prog., 48:141-173 (1952).

[32] Pope, S. B., Turbulent Flows, Cambridge University Press, Cambridge, UK, 2000.

[33] Rogallo, R. S., Numerical Experiments in Homogeneous Turbulence, NASA TM 81315, 1981.

[34] Batchelor, G. K. and Proudman, I., The Large-Scale Structure of Homogeneous Turbulence, Phil. Trans. Royal Soc. London, 248(949):46-405 (1956).

[35] Riley, J. J. and Patterson, G. S., Diffusion Experiments with Numerically Integrated Isotropic Turbulence, Phys. Fluids, 17:292-297 (1974).

[36] Squires, K. D. and Eaton, J. K., Preferential Concentration of Particles by Turbulence, Phys. Fluids, 3(5):1169-1178 (1991).

[37] Wang, L.-P. and Maxey, M. R., Settling Velocity and Concentration Distribution of Heavy Particles in Isotropic Turbulence, J. Fluid Mech., 256:27-68 (1993).

[38] Mashayek, F., Jaberi, F. A., Miller, R. S., and Givi, P., Dispersion and Polydispersity of Droplets in Stationary Isotropic Turbulence, Int. J. Multiphase Flow, 23(2):337-355 (1997). 
[39] Sundaram, S. and Collins, L. R., Collision Statistics in an Isotropic ParticleLaden Turbulent Suspension. Part 1. Direct Numerical Simulations, J. Fluid Mech., 335:75-109 (1997).

[40] Holtzer, G. L. and Collins, L. R., Relationship Between the Intrinsic Radial Distribution Function for an Isotropic Field of Particles and Lower-dimensional Measurements, J. Fluid Mech., 459:93-102 (2002).

[41] Collins, L. R. and Keswani, A., Reynolds Number Scaling of Particle Clustering in Turbulent Aerosols, New Journal of Physics, 119:Art. No. 119 (2004).

[42] Chun, J., Koch, D. L., Rani, S. L., Ahluwalia, A., and Collins, L. R., Clustering of Aerosol Particles in Isotropic Turbulence, J. Fluid Mech., 536:219-251 (2005).

[43] Shotorban, B. and Balachandar, S., Particle Concentration in Homogeneous Shear Turbulence Simulated via Lagrangian and Equilibrium Eulerian Approaches, Phys. Fluids, 18 (2006).

[44] Reade, W. C. and Collins, L. R., A Numerical Study of the Particle Size Distribution of an Aerosol Undergoing Turbulent Coagulation, J. Fluid Mech., 415:45-64 (2000).

[45] Squires, K. D. and Eaton, J. K., Particle Response and Turbulence Modification in Isotropic Turbulence, Phys. Fluids, 2:1191-1203 (1990).

[46] Boivin, M., Simonin, O., and Squires, K. D., Direct Numerical Simulation of Turbulence Modulation by Particles in Isotropic Turbulence, J. Fluid Mech., 375:235-263 (1998).

[47] Barré, C., Mashayek, F., and Taulbee, D. B., Statistics in Particle-Laden Plane Strain Turbulence by Direct Numerical Simulation, Int. J. Multiphase Flow, 27(2):347-378 (2001).

[48] Sengupta, K., Russell, K., Minkowycz, W. J., and Mashayek, F., Numerical Simulation Data for Assessment of Particle-Laden Turbulent Flow Models, Int. J. Heat Mass Transfer, 48(15):3035-3046 (2005).

[49] Taulbee, D. B., Mashayek, F., and Barré, C., Simulation and Reynolds Stress Modeling of Particle-Laden Turbulent Shear Flows, Int. J. Heat Fluid Flow, 20(4):368-373 (1999).

[50] Mashayek, F. and Taulbee, D. B., Turbulent Gas-Solid Flows. Part I: Direct Simulations and Reynolds Stress Closures, Numer. Heat Transfer, Part B, 41(1):1-29 (2002).

[51] Jaberi, F. A., Temperature Fluctuations in Particle-Laden Homogeneous Turbulent Flows, Int. J. Heat Mass Transfer, 41:4081-4093 (1998).

[52] Jaberi, F. A. and Mashayek, F., Temperature Decay in Two-Phase Turbulent Flows, Int. J. Heat Mass Transfer, 43(6):993-1005 (2000). 
[53] Sato, Y., Deutsch, E., and Simonin, O., Direct Numerical Simulations of Heat Transfer by Solid Particles Suspended in Homogeneous Isotropic Turbulence, Int. J. Heat Fluid Flow, 19:187-192 (1998).

[54] Shotorban, B., Mashayek, F., and Pandya, R. V. R., Temperature Statistics in Particle-Laden Turbulent Homogeneous Shear Flow, Int. J. Multiphase Flow, 29(8):1333-1353 (2003).

[55] Pandya, R. V. R. and Mashayek, F., Non-Isothermal Dispersed Phase of Particles in Turbulent Flow, J. Fluid Mech., 475:205-245 (2003).

[56] Mashayek, F., Droplet-Turbulence Interactions in Low-Mach-Number Homogeneous Shear Two-Phase Flows, J. Fluid Mech., 367:163-203 (1998).

[57] Mashayek, F., Direct Numerical Simulations of Evaporating Droplet Dispersion in Forced Low Mach Number Turbulence, Int. J. Heat Mass Transfer, 41(17):2601-2617 (1998).

[58] Mashayek, F., Simulations of Reacting Droplets Dispersed in Isotropic Turbulence, AIAA J., 37(11):1420-1425 (1999).

[59] Mashayek, F., Numerical Investigation of Reacting Droplets in Homogeneous Shear Turbulence, J. Fluid Mech., 405:1-36 (2000).

[60] Mashayek, F. and Jacobs, G. B., Temperature-Dependent Reaction in DropletLaden Homogeneous Turbulence, Numer. Heat Transfer, Part A, 39:101-121 (2001).

[61] Mashayek, F., Velocity and Temperature Statistics in Reacting Droplet-Laden Homogeneous Shear Turbulence, J. Propulsion Power, 17(1):197-202 (2001), also appeared as AIAA Paper 2000-0183.

[62] Yeh, F. and Lei, U., On the Motion of Small Particles in a Homogeneous Isotropic Turbulent Flow, Phys. Fluids, 3(11):2571-2586 (1991).

[63] Yeh, F. and Lei, U., On the Motion of Small Particles in a Homogeneous Turbulent Shear Flow, Phys. Fluids, 3(11):2758-2776 (1991).

[64] Simonin, O., Deutsch, E., and Minier, J. P., Eulerian Prediction of the Fluid/Particle Correlated Motion in Turbulent Two-Phase Flows, Appl. Sci. Res., 51:275-283 (1993).

[65] Boivin, M., Simonin, O., and Squires, K. D., On the Prediction of Gas-Solid Flows with Two-Way Coupling Using Large Eddy Simulation, Phys. Fluids, 12:2080-2090 (2000).

[66] Armenio, V., Piomelli, U., and Fiorotto, V., Effect of the Subgrid Scales on Particles Motion, Phys. Fluids, 11:3030-3042 (1999).

[67] Kuerten, J. G. M. and Verman, A. W., Can Turbophoresis Be Predicted by Large-Eddy Simulation?, Phys. Fluids, 17:011701 (2005). 
[68] Shotorban, B., Modeling of Subgrid-scale Effects on Particles in Large-eddy Simulation of Turbulent Two-phase Flows, Ph.D. Thesis, Universtiy of Illinois at Chicago, Chicago, IL, 2005.

[69] Shotorban, B. and Mashayek, F., Modeling of Subgrid-scale Effects on Particles by Approximate Deconvolution, Phys. Fluids, 17(8) (2005).

[70] Kuerten, J. G. M., Sub-grid modeling in particle-laden channel flow, Physics of Fluid, 18(5):1207-1223 (2006).

[71] Shotorban, B., Zhang, K. K. Q., and Mashayek, F., Improvement of Particle Concentration Prediction in Large-Eddy Simulation by Defiltering, Int. J. Heat Mass Transfer, 50:3728-3739 (2007).

[72] Stolz, S., Adams, N. A., and Kleiser, L., An Approximate Deconvolution for Large-Eddy Simulation with Application to Incompressible Wall-Bounded Flows, Phys. Fluids, 13:997 (2001).

[73] Shotorban, B. and Mashayek, F., A Stochastic Model for Particle Motion in Large-Eddy Simulation, J. Turbulence, 18(7):1-13 (2006).

[74] Pozorski, J. and Minier, J., On the Lagrangian Turbulent Dispersion Models Based on the Langevin Equation, Int. J. Multiphase Flow, 24:913-945 (1998).

[75] Pozorski, J. and Minier, J. P., Probability Density Function Modeling of Dispersed Two-Phase Turbulent Flows, Phys. Rev. E, 59:855-863 (1999).

[76] Minier, J.-P. and Peirano, E., The PDF Approach to Turbulent Polydispersed Two-Phase Flows, Physics Reports, 352:1-214 (2001).

[77] Berrouk, A. S., Laurence, D., Riley, J. J., and Stock, D. E., Stochastic Modelling of Inertial Particle Dispersion by Subgrid Motion for LES of High Reynolds Number Pipe flow, J. Turbulence, 50 (2007).

[78] Giscquel, L. Y. M., Givi, P., Jaberi, F. A., and Pope, S. B., Velocity Filtered Density Function for Large-Eddy Simulation of Turbulent Flows, Phys. Fluids, 14(3):1196-1213 (2002).

[79] Shotorban, B. and Mashayek, F., On Stochastic Modeling of Heavy Particle Dispersion in LES of Two-Phase Turbulent Flows, in Proceedings of the IUTAM Symposium on Computational Approaches to Disperse Multiphase Flow, Kluwer, 2005.

[80] Jacobs, G. B., Kopriva, D. A., and Mashayek, F., Validation Study of a Multidomain Spectral Code for Simulation of Turbulent Flows, AIAA J., 43(6):1256-1264 (2005).

[81] Kopriva, D. A., A Staggered-Grid Multidomain Spectral Method for the Compressible Navier-Stokes Equations., J. Comp. Phys., 244:142-158 (1998).

[82] Jacobs, G. B., Kopriva, D. A., and Mashayek, F., A Comparison of Outflow Boundary Conditions for the Multidomain Staggered-Grid Spectral Method, Numer. Heat Transfer, Part B, 44(3):225-251 (2003). 
[83] Jacobs, G. B., Kopriva, D. A., and Mashayek, F., Compressible subsonic particle-laden flow over a square cylinder, J. Propulsion and Power, 20(2):353359 (2004).

[84] Jacobs, G. B., Kopriva, D. A., and Mashayek, F., A conservative isothermal wall boundary condition for the compressible Navier-Stokes equations, J. Sci. Comput., 30(2):177-192 (2005).

[85] Jacobs, G. B., Numerical Simulation of Two-Phase Turbulent Compressible Flows with a Multidomain Spectral Method, Ph.D. Thesis, University of Illinois at Chicago, Chicago, IL, 2003.

[86] Sengupta, K., Mashayek, F., and Jacobs, G., Direct simulation of turbulent flows using spectral methods, AIAA Paper 2008-1450, 2008.

[87] Crowe, C. T., Sharma, M. P., and Stock, D. E., The Particle-Source in Cell (PSI-Cell) Model for Gas-Droplet Flows, J. Fluids Eng., 6:325-332 (1977).

[88] Jacobs, G. B., Kopriva, D. A., and Mashayek, F., Towards efficient tracking of inertial particles with high-order multidomain methods, J. Comp. Appl. Math., 206:392-408 (2007).

[89] Jacobs, G. B. and Hesthaven, J. S., High-order Nodal Discontinuous Galerkin Particle-in-cell Method on Unstructured Grids., J. Comp. Phys., 214:96-121 (2006).

[90] Maxey, M. R., Patel, B. K., and Wang, L., Simulations of Dispersed Turbulent Multiphase Flow, Fluid Dyn. Res., 20:143-156 (1997).

[91] Jacobs, G., Lapenta, G., and Hesthaven, J., Simulations of plasmas with a high-order discontinuous Galerkin particle-in-cell solver, AIAA Paper 2006$1171,2006$.

[92] Armstrong, K., How does inertial particle dispersion relate to the finite time Lyapunov exponent in a vortex dominated wake?, Master's thesis, San Diego State University, 2008.

[93] Jacobs, G., Inertial particle behavior in a separated, turbulent flow, AIAA Paper 2008-1159, 2008.

[94] Le, H. and Moin, P., Direct Simulations of Turbulent Flow over a BackwardFacing Step, Department of Mechanical Engineering Report TF-58, Stanford University, Stanford, CA, 1994.

[95] Surana, A. and Haller, G., Ghost manifolds in slow-fast systems, with application to unsteady fluid flow separation, Physica D, (1007), in press.

[96] Weldon, M., Peacock, T., Jacobs, G., Helu, M., and Haller, G., Experimental and numerical investigation of the kinematic theory of unsteady separation, J. Fluid Mech., (2008), to appear. 
[97] Wengle, H., Huppertz, A., Barwolff, G., and Janke, G., The Manipulated Transitional Backward-Facing Step Flow: An Experimental and Direct Numerical Simulation Investigation, Eur. J. Mech. B - Fluids, 20:25-46 (2001).

[98] Roy, G. D. and Mashayek, F., editors, Proceedings of the Fourteenth ONR Propulsion Meeting, University of Illinois at Chicago, Chicago, IL, 2001.

[99] Levin, J. G., Iskandarani, M., and Haidvogel, D. B., A spectral filtering procedure for eddy-resolving simulations with a spectral element ocean model, Journal of Computational Physics, 137:130-154 (1997).

[100] Fischer, P. F. and Mullen, J. S., Filter based stabilization of spectral element methods, Comptes Rendus a l'Academie des Sciences Paris, Ser. 1, Anal. Numer, 332:265-270 (2001).

[101] Karamanos, G. S., Large-eddy simulation using unstructured spectral/hp finite elements, Ph.D. Thesis, Imperial College of Science and Technology, London, UK, 1999.

[102] Karamanos, G.-S. and Karniadakis, G. E., A spectral vanishing viscosity method for large-eddy simulations, Journal of Comput. Physics, 163:22-50 (2000).

[103] Wang, Q. and Squires, K., Large Eddy Simulation of Particle-Laden Turbulent Channel Flow, Phys. Fluids, 8(5):1207-1223 (1996).

[104] Rouson, D. W. I. and Eaton, J. K., On the Preferential Concentration of Solid Particles in Turbulent Channel Flow, J. Fluid Mech., 428:149-169 (2001).

[105] Kulick, J. D., Fessler, J. R., and Eaton, J. K., Particle Response and Turbulence Modification in Fully Developed Channel Flow, J. Fluid Mech., 277:109-134 (1994).

[106] Moser, R., Kim, J., and Mansour, N. N., Direct Numerical Simulation of Turbulent Channel Flow up to $R e_{\tau}=590$, Phys. Fluids, 11:943-945 (1999).

[107] Niederschulte, M. A., Adrian, R. J., and Hanratty, T. J., Measurements of turbulent flow in a channel at low Reynolds numbers, Exp. Fluids, 9:222-230 (1990). 


\section{Figure captions}

Figure 1. Particle accumulation in the DNS of a homogeneous shear turbulent flow for various Stokes numbers in one-way coupling at the same realization of the carrier-phase. Stokes number here is defined as the ratio of the particle time constant and the instantaneous Kolmogorov time scale. Particles are projected from a thin slice perpendicular to the spanwise direction. Simulation details are given in [71].

Figure 2. 2D (1,2, and 3 show the streamwise, cross-stream, and spanwise directions, respectively.) and 3D Radial Distribution Functions in homogeneous shear turbulence for $S t=1.2$. Stokes number here is defined as the ratio of the particle time constant and the instantaneous Kolmogorov time scale. Simulation details are given in [71].

Figure 3. Snapshots of particles with $S t=5.0$ periodically injected upstream and downstream of the square cylinder. The square cylinder flow at a Reynolds number, $R e=150$ and free stream Mach number, $M a_{\infty}=0.1$ sheds periodically as shown by the vorticity contours and streamlines. The particles injected downstream of the square cylinder accumulate at the square corner and shed in packages.

Figure 4. Computational domain and grid for simulation of the flow over a backward-facing step.

Figure 5. Instantaneous (a) and averaged (b) streamlines of the 2-D flow over an open backward-facing step at Reynolds number $R e_{f}=1500$ and Mach number $M a=0.4$. The instantaneous streamlines show a complex vortex shedding pattern of this flow. If averaged, the streamline pattern exhibits a typical recirculation with edge and corner vortex separation, and a shear layer reattachment.

Figure 6. Inertial particles and vorticity contours at three equi-spaced consecutive times within a flow period in the quasi-steady backward-facing step flow. The particles are continuously injected near the corner separation location. Material lines form at the corner separation location and are anchored in the fixed averaged zero skin friction location.

Figure 7. Comparison of the Favre averaged $\tilde{u}(z)$-velocity profiles at various $x$ locations behind a backward-facing step for compressible flow spectral element computations with a sixth, $p=6$, and eight, $p=8$, order accuracy to the Reynolds average $\bar{u}(z)$-velocity profiles of the incompressible flow experiment [97] for a backward-facing step flow at $R e=3000$.

Figure 8. Comparison of Favre averaged Reynolds stress component, $-\widetilde{u^{\prime \prime} w^{\prime \prime}}$, 
plotted versus $z$ at different $x$ locations behind the backward-facing step for compressible flow spectral element computations with $p=8$ and the incompressible flow experimental results in [97] at $R e=3000$.

Figure 9. Schematic of the particle injection locations for the backward-facing step flow.

Figure 10. Side view snapshot of the pressure iso-contours for the backwardfacing step flow with (a-e) and without (f-j) counter current shear. Side view snapshots of the particle distribution for injection locations outside the boundary layer (b, d, g, i) and inside the boundary layer (c, e, h, j) for $\mathrm{St}=1(\mathrm{~b}, \mathrm{c}, \mathrm{g}, \mathrm{h})$ and $S t=0.1(\mathrm{~d}, \mathrm{e}, \mathrm{i}, \mathrm{j})$.

Figure 11. Top view snapshot of particle-laden flow in Fig. 10.

Figure 12. Mean normalized streamwise velocity plotted in wall coordinates for channel flow at $\mathrm{Re}=3000$.

Figure 13. Dispersed phase mean normalized streamwise velocity plotted in wall coordinates for the channel flow at $\mathrm{Re}=3000$. 

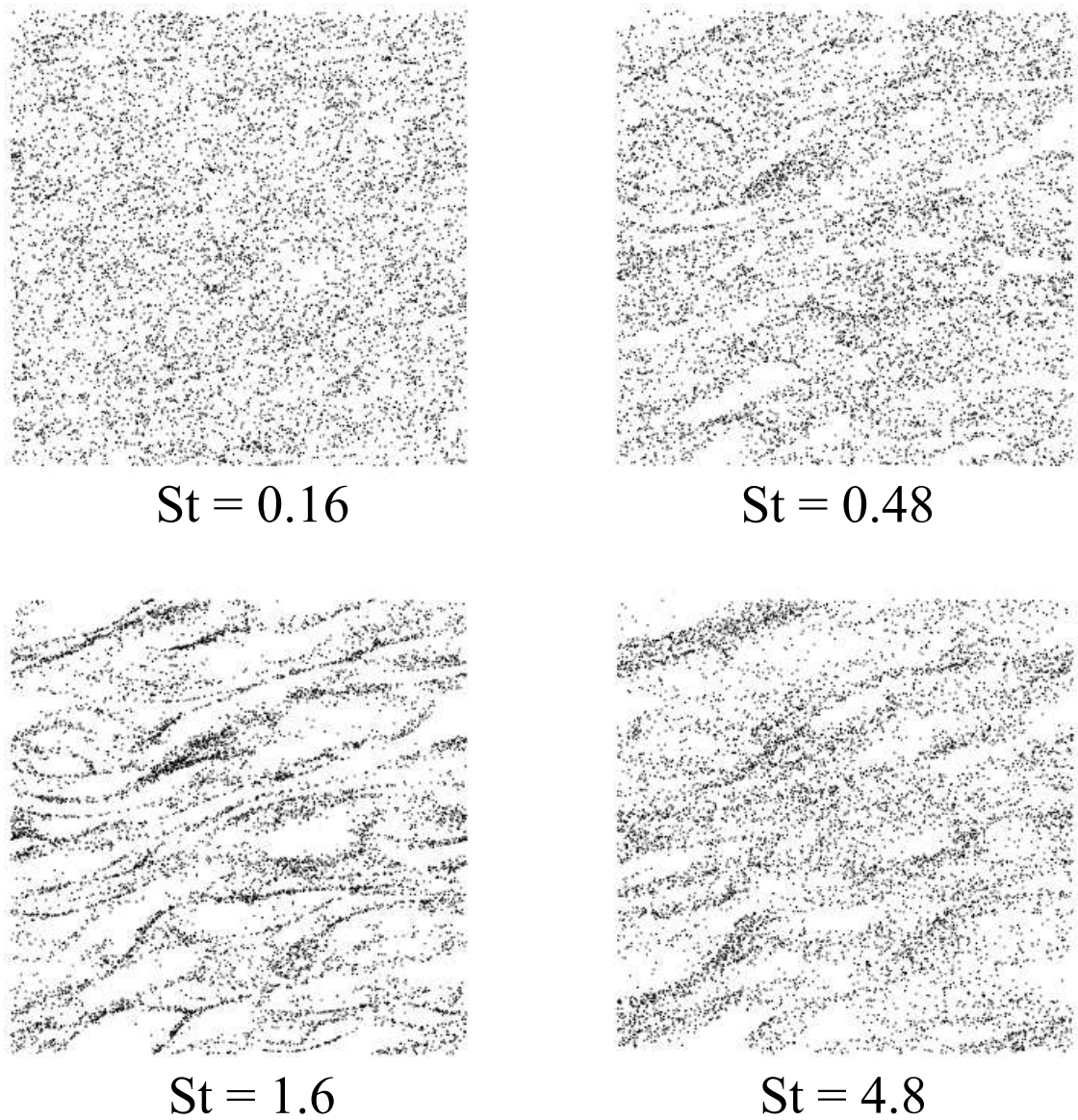

Fig. 1. Particle accumulation in the DNS of a homogeneous shear turbulent flow for various Stokes numbers in one-way coupling at the same realization of the carrier-phase. Stokes number here is defined as the ratio of the particle time constant and the instantaneous Kolmogorov time scale. Particles are projected from a thin slice perpendicular to the spanwise direction. Simulation details are given in [71]. 


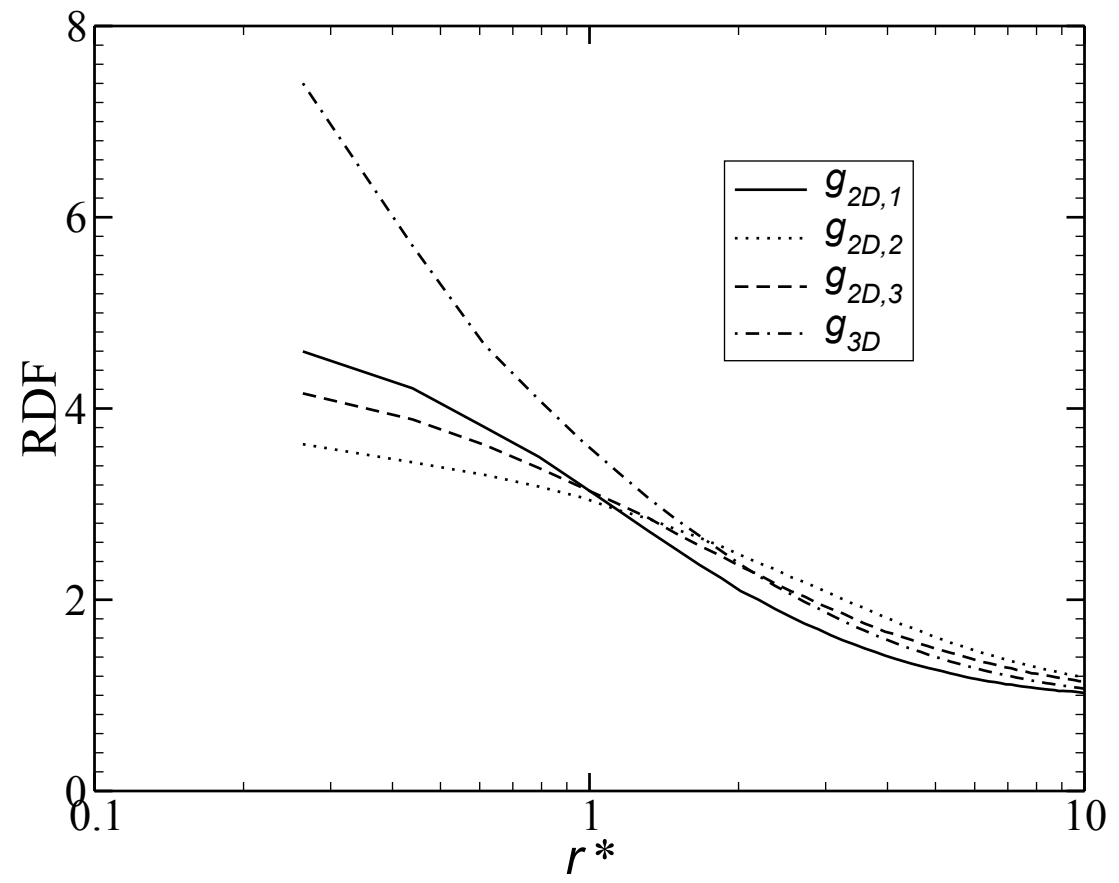

Fig. 2. 2D (1,2, and 3 show the streamwise, cross-stream, and spanwise directions, respectively.) and 3D Radial Distribution Functions in homogeneous shear turbulence for $S t=1.2$. Stokes number here is defined as the ratio of the particle time constant and the instantaneous Kolmogorov time scale. Simulation details are given in [71]. 


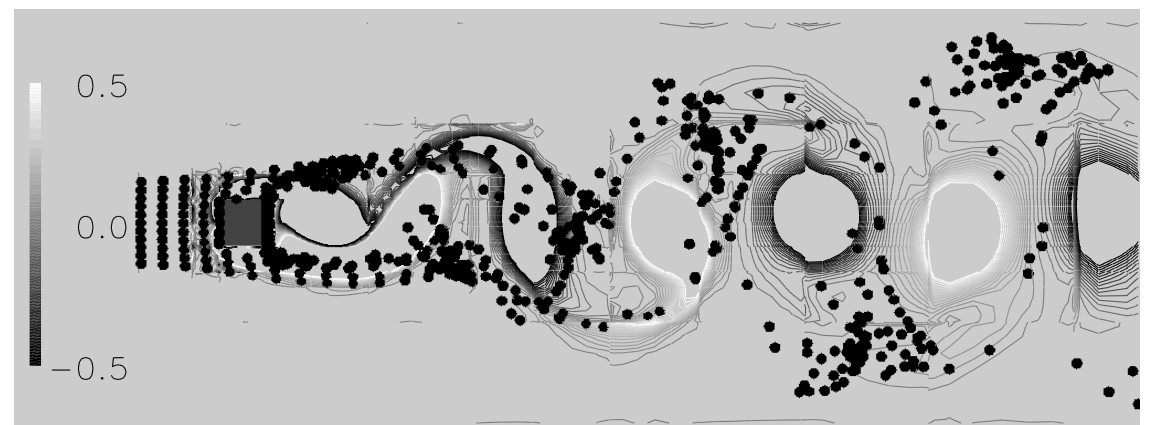

Fig. 3. Snapshots of particles with $S t=5.0$ periodically injected upstream and downstream of the square cylinder. The square cylinder flow at a Reynolds number, $R e=150$ and free stream Mach number, $M a_{\infty}=0.1$ sheds periodically as shown by the vorticity contours and streamlines. The particles injected downstream of the square cylinder accumulate at the square corner and shed in packages. 


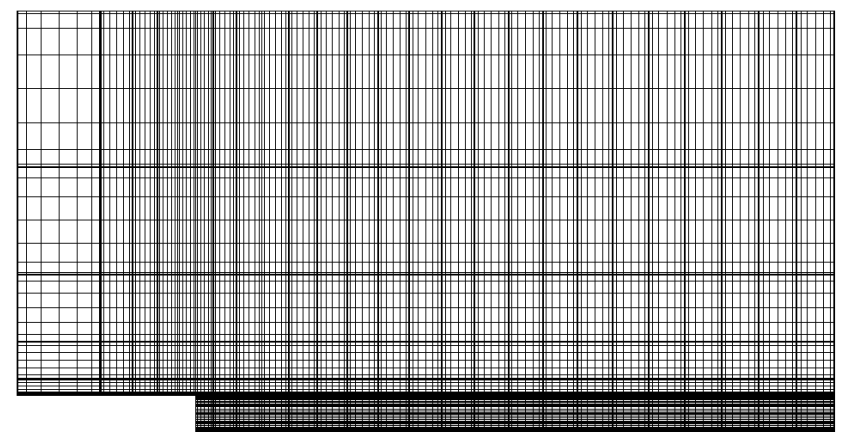

Fig. 4. Computational domain and grid for simulation of the flow over a backwardfacing step. 
(a) streamline snapshot

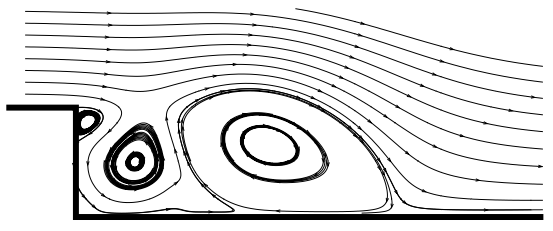

(b) averaged streamlines

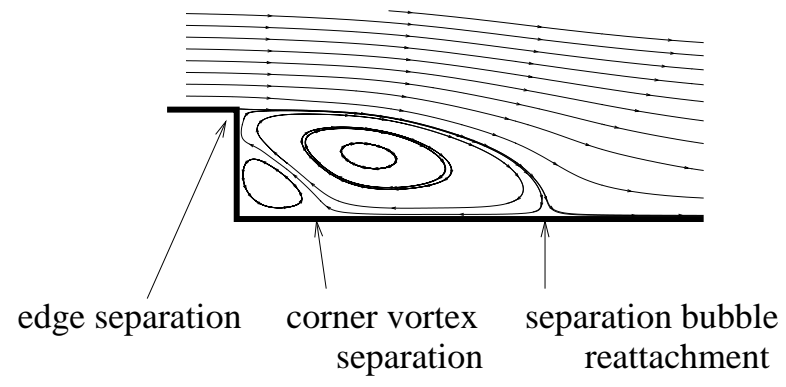

Fig. 5. Instantaneous (a) and averaged (b) streamlines of the 2-D flow over an open backward-facing step at Reynolds number $R e_{f}=1500$ and Mach number $M a=0.4$. The instantaneous streamlines show a complex vortex shedding pattern of this flow. If averaged, the streamline pattern exhibits a typical recirculation with edge and corner vortex separation, and a shear layer reattachment. 


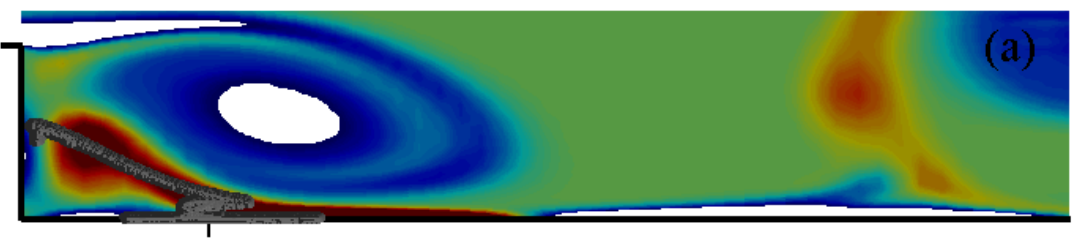

corner separation

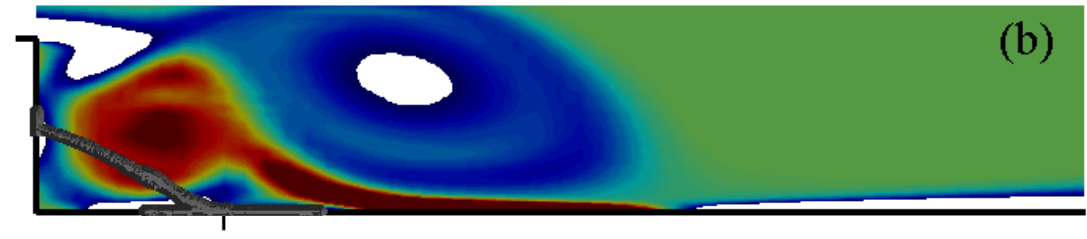

corner separation

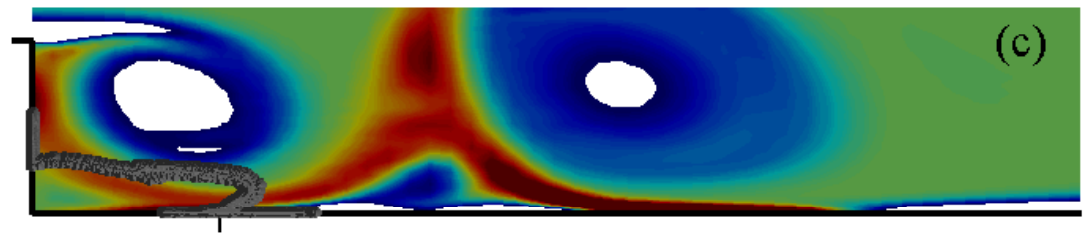

corner separation

Fig. 6. Inertial particles and vorticity contours at three equi-spaced consecutive times within a flow period in the quasi-steady backward-facing step flow. The particles are continuously injected near the corner separation location. Material lines form at the corner separation location and are anchored in the fixed averaged zero skin friction location. 


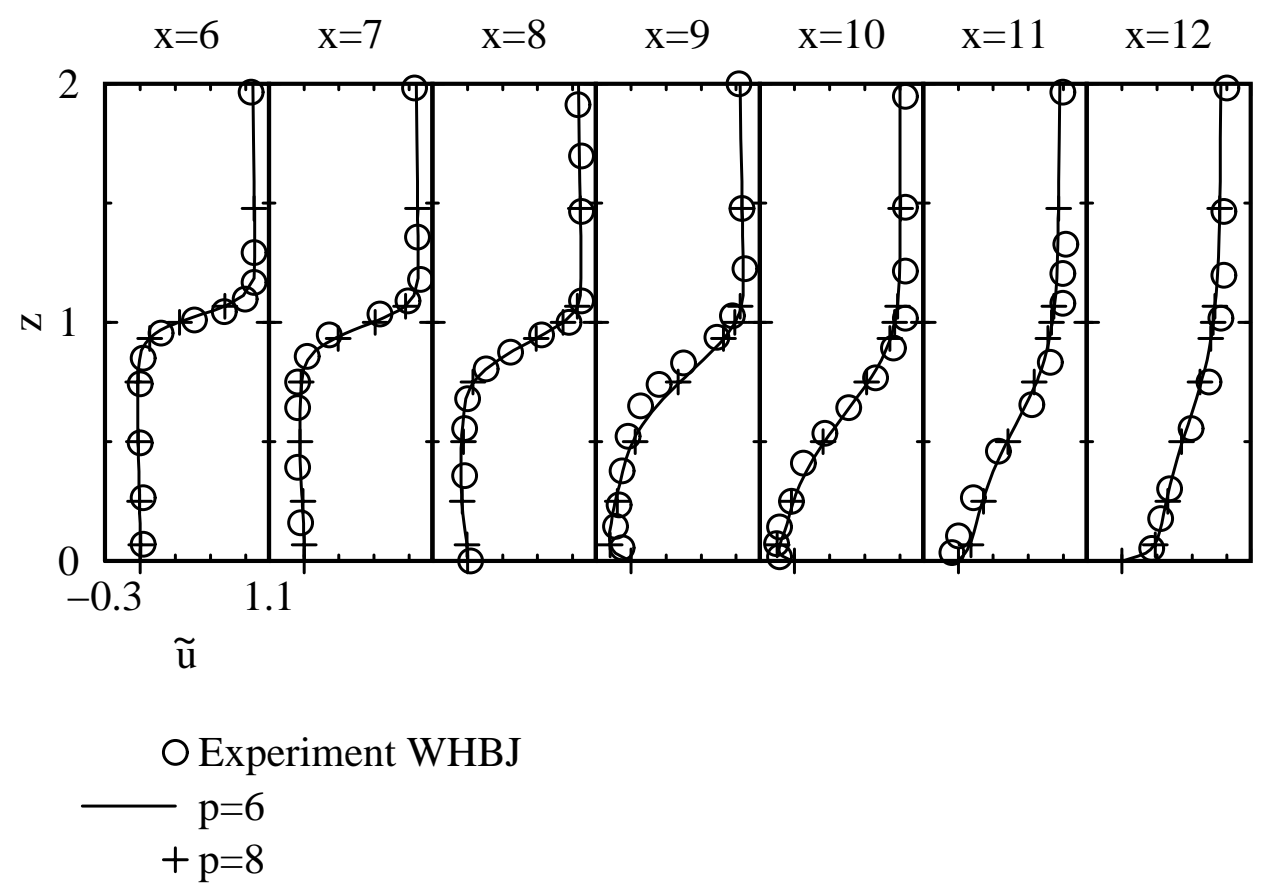

Fig. 7. Comparison of the Favre averaged $\tilde{u}(z)$-velocity profiles at various $x$ locations behind a backward-facing step for compressible flow spectral element computations with a sixth, $p=6$, and eight, $p=8$, order accuracy to the Reynolds average $\bar{u}(z)$-velocity profiles of the incompressible flow experiment [97] for a backward-facing step flow at $R e=3000$. 

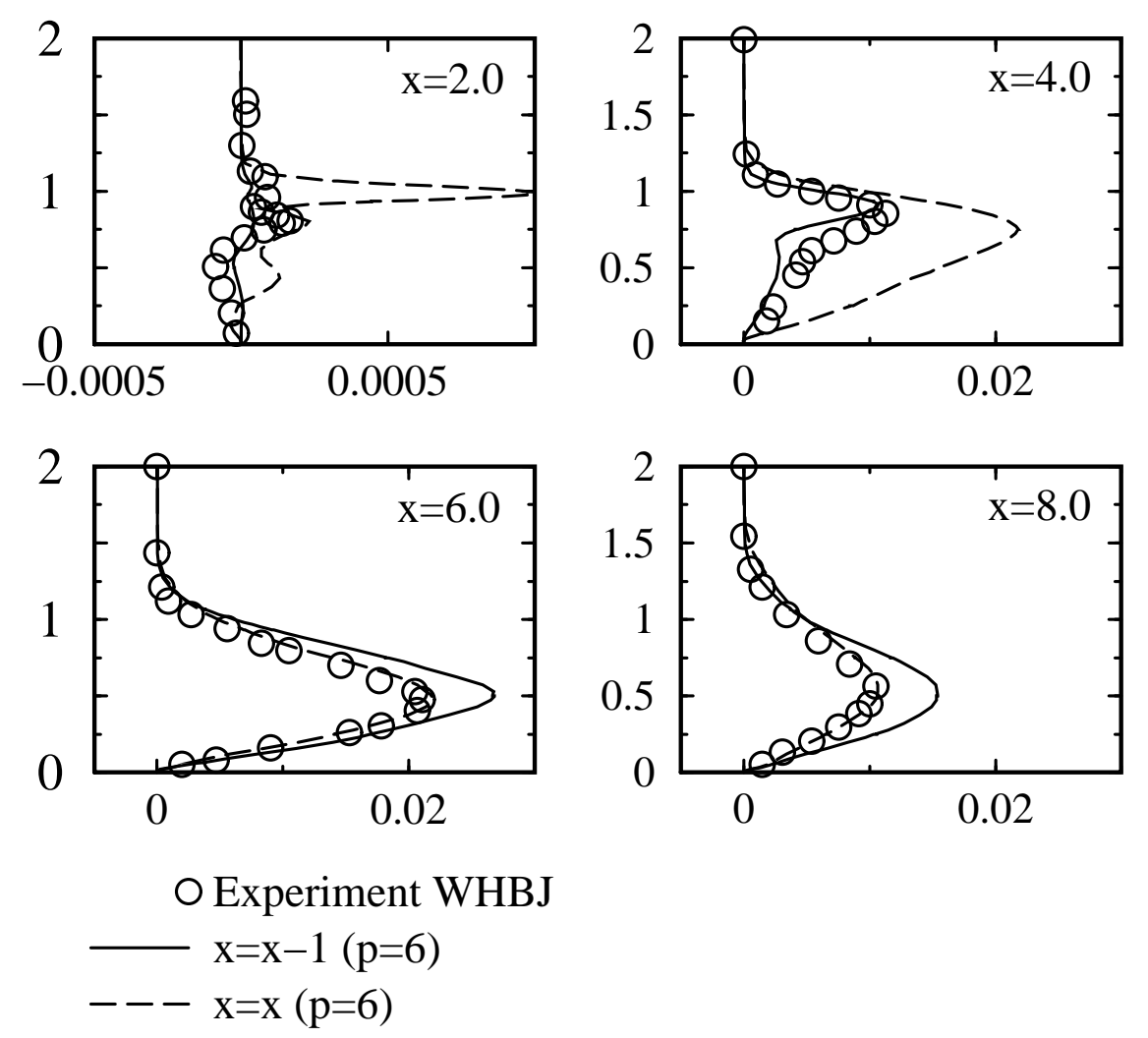

Fig. 8. Comparison of Favre averaged Reynolds stress component, $-\widetilde{u^{\prime \prime} w^{\prime \prime}}$, plotted versus $z$ at different $x$ locations behind the backward-facing step for compressible flow spectral element computations with $p=8$ and the incompressible flow experimental results in [97] at $R e=3000$. 


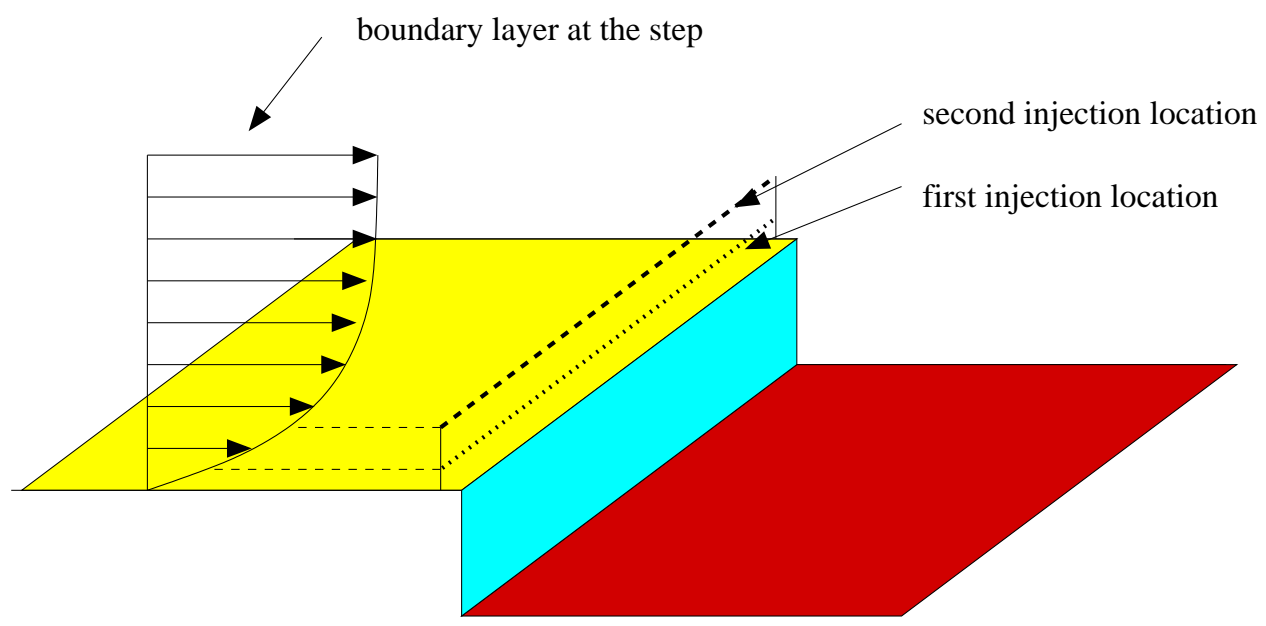

Fig. 9. Schematic of the particle injection locations for the backward-facing step flow. 


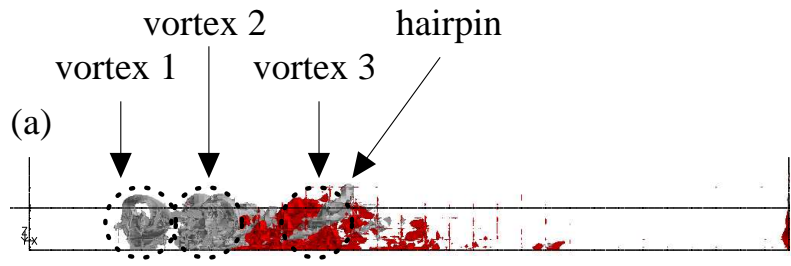

(b)

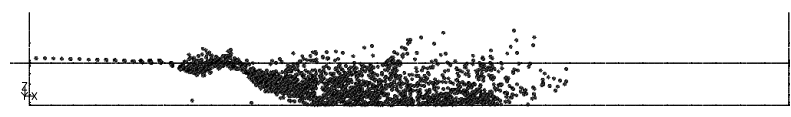

(c)

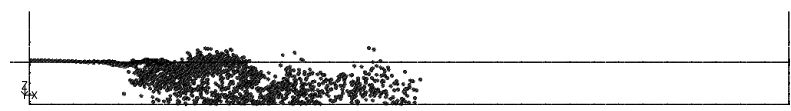

(d)

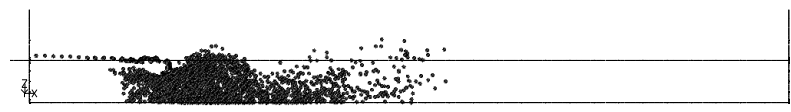

(e)

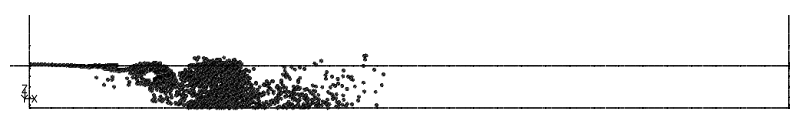

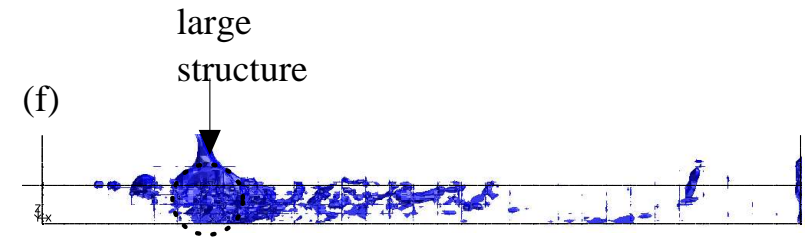

$(\mathrm{g})$

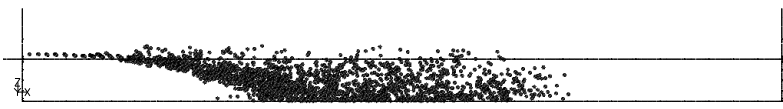

(h)

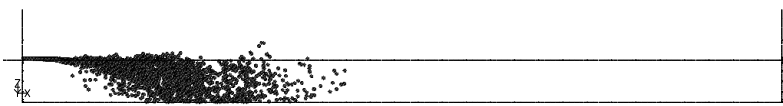

(i)

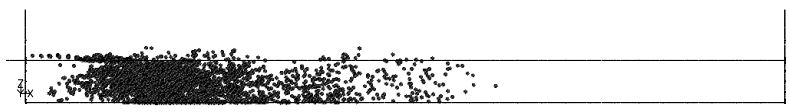

(j)

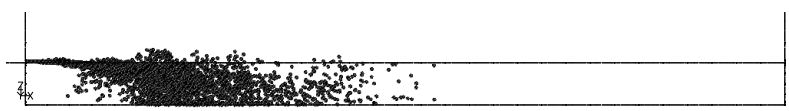

Fig. 10. Side view snapshot of the pressure iso-contours for the backward-facing step flow with (a-e) and without (f-j) counter current shear. Side view snapshots of the particle distribution for injection locations outside the boundary layer (b, d, g, i) and inside the boundary layer (c, e, h, j) for $\mathrm{St}=1(\mathrm{~b}, \mathrm{c}, \mathrm{g}, \mathrm{h})$ and $S t=0.1(\mathrm{~d}, \mathrm{e}, \mathrm{i}, \mathrm{j})$. 

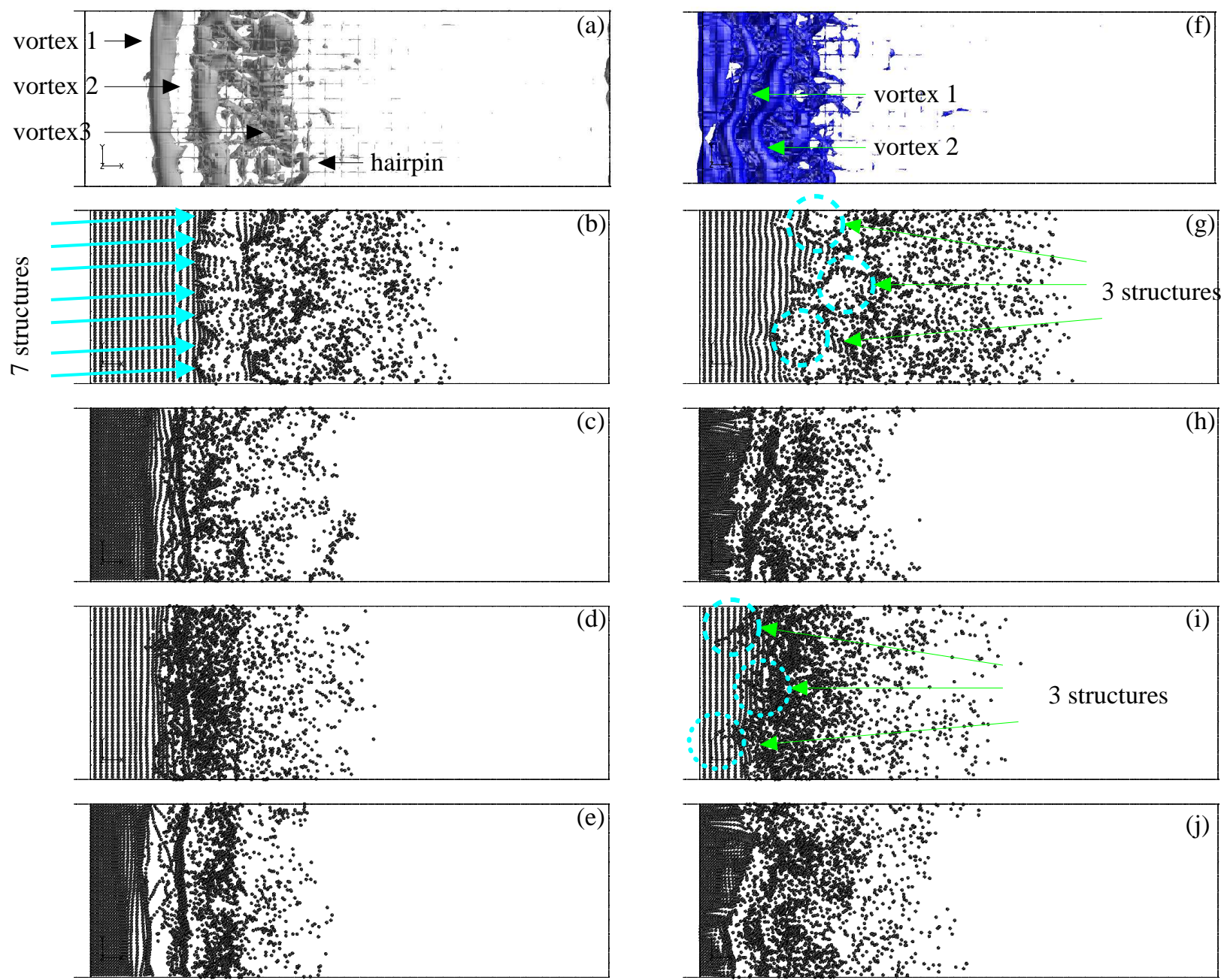

Fig. 11. Top view snapshot of particle-laden flow in Fig. 10. 


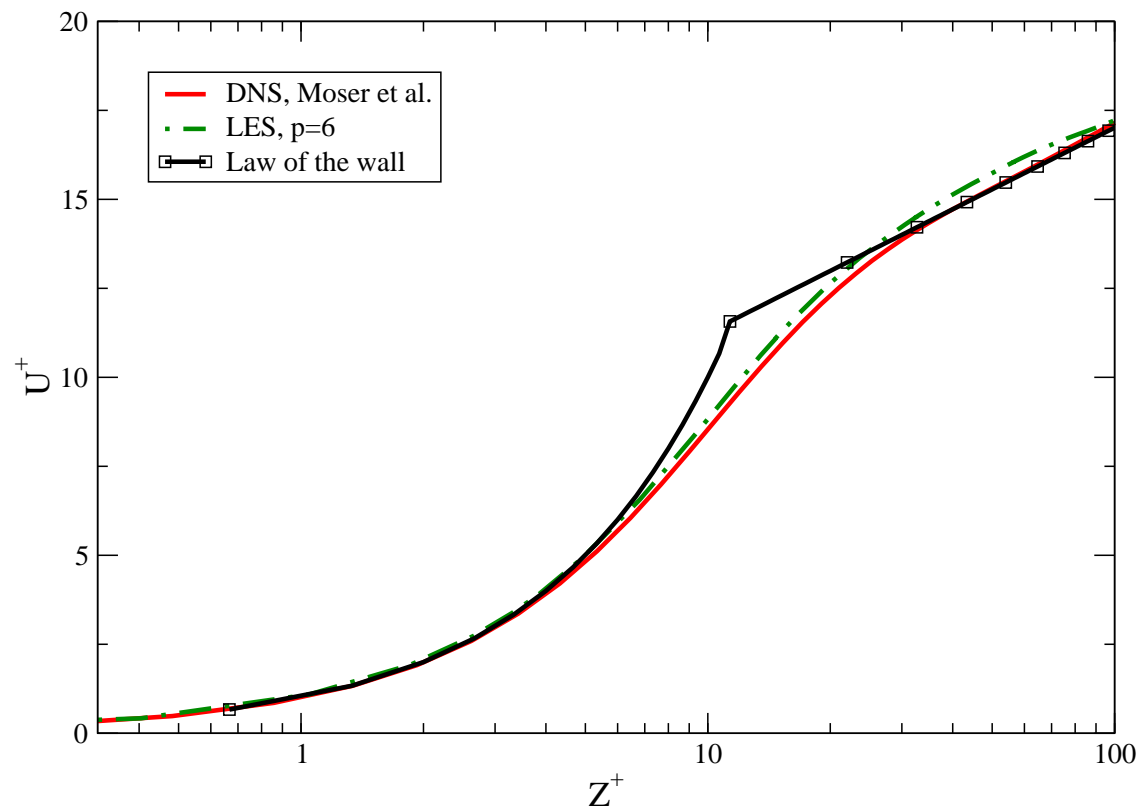

Fig. 12. Mean normalized streamwise velocity plotted in wall coordinates for channel flow at $\operatorname{Re}=3000$. 


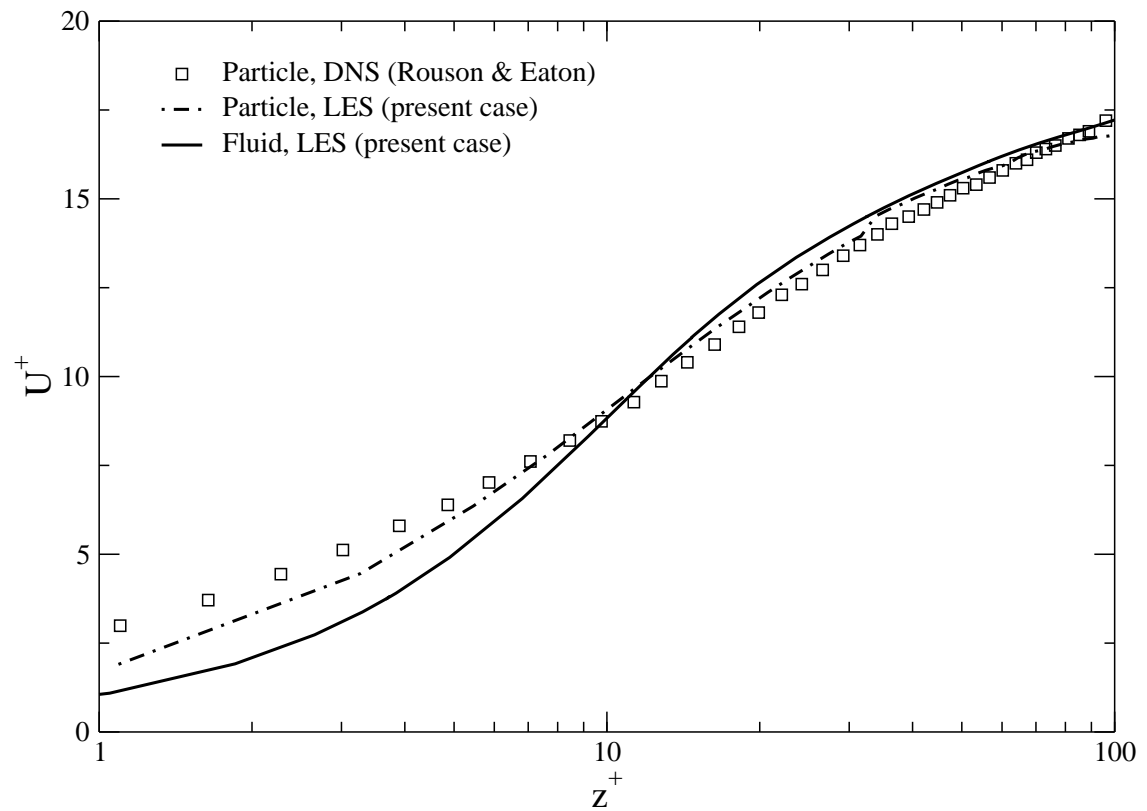

Fig. 13. Dispersed phase mean normalized streamwise velocity plotted in wall coordinates for the channel flow at $\mathrm{Re}=3000$. 